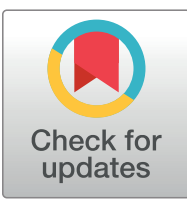

\section{G openaccess}

Citation: Rivas MA, Avila BE, Koskela J, Huang $\mathrm{H}$, Stevens C, Pirinen M, et al. (2018) Insights into the genetic epidemiology of Crohn's and rare diseases in the Ashkenazi Jewish population. PLoS Genet 14 (5): e1007329. https://doi.org/10.1371/journal. pgen.1007329

Editor: Scott M. Williams, Case Western Reserve University School of Medicine, UNITED STATES

Received: November 9, 2017

Accepted: March 22, 2018

Published: May 24, 2018

Copyright: ๑ 2018 Rivas et al. This is an open access article distributed under the terms of the Creative Commons Attribution License, which permits unrestricted use, distribution, and reproduction in any medium, provided the original author and source are credited.

Data Availability Statement: All data are published on dbGap and are available via the following link: https://www.ncbi.nlm.nih.gov/projects/gap/cgi-bin/ study.cgi?study_id=phs001076.v1.p1

Funding: This study is supported in part by The Leona M. \& Harry B. Helmsley Charitable Trust (www.helmsleytrust.org) [2015PG-IBD001] and the Large Scale Sequencing Grant from the US National Institutes of Health (www.nih.gov) [5 U54 HG003067-13]. The funders had no role in study

\title{
Insights into the genetic epidemiology of Crohn's and rare diseases in the Ashkenazi Jewish population
}

\begin{abstract}
Manuel A. Rivas ${ }^{1,2 *}$, Brandon E. Avila ${ }^{1,3}$, Jukka Koskela ${ }^{1,3,4}$, Hailiang Huang ${ }^{1,3}$, Christine Stevens ${ }^{1}$, Matti Pirinen ${ }^{4,5}$, Talin Haritunians ${ }^{6}$, Benjamin M. Neale ${ }^{1,3}$, Mitja Kurki $^{1,3}$, Andrea Ganna ${ }^{1,3}$, Daniel Graham ${ }^{1}$, Benjamin Glaser ${ }^{7}$, Inga Peter ${ }^{8}$, Gil Atzmon ${ }^{9,10}$, Nir Barzilai ${ }^{9}$, Adam P. Levine ${ }^{11}$, Elena Schiff ${ }^{11}$, Nikolas Pontikos ${ }^{11,12}$, Ben Weisburd ${ }^{1,3}$, Monkol Lek ${ }^{1,3}$, Konrad J. Karczewski ${ }^{1,3}$, Jonathan Bloom ${ }^{1,3}$, Eric V. Minikel ${ }^{1,3}$, Britt-Sabina Petersen ${ }^{13}$, Laurent Beaugerie ${ }^{14}$, Philippe Seksik ${ }^{14}$, Jacques Cosnes ${ }^{14}$, Stefan Schreiber ${ }^{15}$, Bernd Bokemeyer ${ }^{16}$, Johannes Bethge ${ }^{15}$, International IBD Genetics Consortium", NIDDK IBD Genetics Consortium", T2D-GENES Consortium 71 , Graham Heap ${ }^{17}$, Tariq Ahmad ${ }^{18}$, Vincent Plagnol ${ }^{12}$, Anthony W. Segal ${ }^{11}$, Stephan Targan $^{6}$, Dan Turner ${ }^{19}$, Paivi Saavalainen ${ }^{20}$, Martti Farkkila ${ }^{21}$, Kimmo Kontula $^{22}$, Aarno Palotie ${ }^{1,4,23}$, Steven R. Brant ${ }^{24,25}$, Richard H. Duerr ${ }^{26,27}$, Mark S. Silverberg ${ }^{28}$, John D. Rioux ${ }^{29,30}$, Rinse K. Weersma ${ }^{31}$, Andre Franke ${ }^{13}$, Luke Jostins ${ }^{32}$, Carl A. Anderson ${ }^{33}$, Jeffrey C. Barrett ${ }^{33}$, Daniel G. MacArthur ${ }^{1,3}$, Chaim Jalas ${ }^{34}$, Harry Sokol ${ }^{14}$, Ramnik J. Xavier ${ }^{1,35}$, Ann Pulver ${ }^{36}$, Judy H. Cho ${ }^{37 *}$, Dermot P. B. McGovern ${ }^{6 *}$, Mark J. Daly ${ }^{1,3,4 *}$
\end{abstract}

1 Medical and Population Genetics, Broad Institute, Cambridge, MA, United States of America,

2 Department of Biomedical Data Science, Stanford University, Stanford, CA, United States of America,

3 Analytical and Translational Genetics Unit, Massachusetts General Hospital, Boston, MA, United States of America, 4 Institute for Molecular Medicine Finland (FIMM), University of Helsinki, Helsinki, Finland, 5 Department of Mathematics and Statistics, University of Helsinki, Helsinki, Finland, 6 Translational Genomics Unit, F. Widjaja Foundation Inflammatory Bowel and Immunobiology Research Institute, CedarsSinai Medical Center, Los Angeles, CA, United States of America, 7 Hadassah-Hebrew University Medical Center, Endocrinology and Metabolism Service Department of Internal Medicine, Jerusalem, Israel, 8 Department of Genetics and Genomic Sciences, Icahn School of Medicine at Mount Sinai, New York, NY, United States of America, 9 Department of Genetics and Medicine, Albert Einstein College of Medicine, Bronx, NY, United States of America, 10 Faculty of Natural Sciences, University of Haifa, Haifa, Israel, 11 Division of Medicine, University College London, London, United Kingdom, 12 UCL Genetics Institute, University College London, London, United Kingdom, 13 Institute of Clinical Molecular Biology, ChristianAlbrechts-University of Kiel, Kiel, Germany, 14 Gastroenterology Department, Saint-Antoine Hospital, APHP, UPMC Univ Paris, Paris, France, 15 Department of Internal Medicine, University Hospital SchleswigHolstein, Kiel, Germany, 16 Gastroenterology Practice, Minden, Germany, 17 IBD Pharmacogenetics, Royal Devon and Exeter NHS Trust, Exeter, United Kingdom, 18 Peninsula College of Medicine and Dentistry, Exeter, United Kingdom, 19 Juliet Keidan Institute of Pediatric Gastroenterology and Nutrition, Shaare Zedek Medical Center, The Hebrew University of Jerusalem, Jerusalem, Israel, 20 Research Programs Unit, Immunobiology, and Department of Medical and Clinical Genetics, University of Helsinki, Helsinki, Finland, 21 Department of Medicine, Division of Gastroenterology, Helsinki University Hospital, Helsinki, Finland, 22 Department of Medicine, University of Helsinki and Helsinki University Central Hospital, Helsinki, Finland, 23 Department of Neurology, Massachusetts General Hospital, Boston, MA, United States of America, 24 Meyerhoff Inflammatory Bowel Disease Center, Department of Medicine, School of Medicine, Johns Hopkins University, Baltimore, MD, United States of America, 25 Department of Epidemiology, Bloomberg School of Public Health, Johns Hopkins University, Baltimore, MD, United States of America, 26 Division of Gastroenterology, Hepatology and Nutrition, Department of Medicine, University of Pittsburgh School of Medicine, Pittsburgh, PA, United States of America, 27 Department of Human Genetics, University of Pittsburgh Graduate School of Public Health, Pittsburgh, PA, United States of America, 28 Inflammatory Bowel Disease Centre, Mount Sinai Hospital, Toronto, Ontario, Canada, 29 Research Center, Montreal Heart Institute, Montréal, Québec, Canada, 30 Department of Medicine, Université de Montréal, Montréal, Québec, Canada, 31 Department of Gastroenterology and Hepatology, University Medical Center Groningen, Groningen, The Netherlands, 32 Wellcome Trust Centre for Human Genetics, Oxford University, Oxford, United Kingdom, 33 Wellcome Trust Sanger Institute, Wellcome Trust Genome Campus, Hinxton, United Kingdom, 34 Bonei Olam, Center for Rare Jewish Genetic Disorders, Brooklyn, NY, United States of America, 35 Gastrointestinal Unit and Center for the Study of Inflammatory Bowel Disease and Center for 
design, data collection and analysis, decision to publish, or preparation of the manuscript.

Competing interests: The authors have declared that no competing interests exist.
Computational and Integrative Biology, Massachusetts General Hospital, Harvard Medical School, Boston, MA, United States of America, 36 Department of Psychiatry and Behavioral Sciences, Johns Hopkins University School of Medicine, Baltimore, MD, United States of America, 37 Icahn School of Medicine at Mount Sinai, Dr Henry D. Janowitz Division of Gastroenterology, New York, NY, United States of America

ๆ Consortium members listed in Acknowledgments section.

*mrivas@stanford.edu (MAR); judy.cho@mssm.edu (JHC); Dermot.McGovern@cshs.org (DPBM); mjdaly@atgu.mgh.harvard.edu (MJD)

\section{Abstract}

As part of a broader collaborative network of exome sequencing studies, we developed a jointly called data set of 5,685 Ashkenazi Jewish exomes. We make publicly available a resource of site and allele frequencies, which should serve as a reference for medical genetics in the Ashkenazim (hosted in part at https://ibd.broadinstitute.org, also available in gno$\mathrm{mAD}$ at http://gnomad.broadinstitute.org). We estimate that $34 \%$ of protein-coding alleles present in the Ashkenazi Jewish population at frequencies greater than $0.2 \%$ are significantly more frequent (mean 15 -fold) than their maximum frequency observed in other reference populations. Arising via a well-described founder effect approximately 30 generations ago, this catalog of enriched alleles can contribute to differences in genetic risk and overall prevalence of diseases between populations. As validation we document $148 \mathrm{AJ}$ enriched protein-altering alleles that overlap with "pathogenic" ClinVar alleles (table available at https://github.com/macarthur-lab/clinvar/blob/master/output/clinvar.tsv), including those that account for 10-100 fold differences in prevalence between AJ and non-AJ populations of some rare diseases, especially recessive conditions, including Gaucher disease (GBA, p.Asn409Ser, 8-fold enrichment); Canavan disease (ASPA, p.Glu285Ala, 12-fold enrichment); and Tay-Sachs disease (HEXA, c.1421+1G>C, 27-fold enrichment; p.Tyr427llefsTer5, 12-fold enrichment). We next sought to use this catalog, of well-established relevance to Mendelian disease, to explore Crohn's disease, a common disease with an estimated two to fourfold excess prevalence in AJ. We specifically attempt to evaluate whether strong acting rare alleles, particularly protein-truncating or otherwise large effect-size alleles, enriched by the same founder-effect, contribute excess genetic risk to Crohn's disease in AJ, and find that ten rare genetic risk factors in NOD2 and $L R R K 2$ are enriched in $A J(p<0.005)$, including several novel contributing alleles, show evidence of association to $C D$. Independently, we find that genomewide common variant risk defined by GWAS shows a strong difference between AJ and non-AJ European control population samples ( 0.97 s.d. higher, $\left.p<10^{-16}\right)$. Taken together, the results suggest coordinated selection in AJ population for higher $\mathrm{CD}$ risk alleles in general. The results and approach illustrate the value of exome sequencing data in case-control studies along with reference data sets like ExAC (sites VCF available via FTP at ftp.broadinstitute. org/pub/ExAC_release/release0.3/) to pinpoint genetic variation that contributes to variable disease predisposition across populations.

\section{Author summary}

The Ashkenazim are a people with ancestry in northern-European Jewish groups. A founder effect caused a bottleneck in this population approximately one thousand years 
ago, resulting in a group of enriched alleles in their genetic makeup. A higher documented prevalence of Crohn's Disease in the Ashkenazim indicates that some enriched alleles may confer risk of having this disease. By studying which genes are enriched, and which of these contribute to Crohn's Disease risk, we are better able to understand the genetic architecture of the affected population, and of the disease itself. Further, we are able to develop a resource containing tables of significantly enriched alleles that are known or suspected to contribute to other disease.

\section{Introduction}

Genetic population isolates like the Ashkenazim, Jews who trace their ancestry to eleventh century central European Jewish groups[1], have previously facilitated the mapping of alleles contributing to human disease predisposition[2-5]. The documented 2-4 fold enrichment of Crohn's Disease (CD) prevalence in the Ashkenazi Jewish (AJ) population[6,7] motivated the use of exome sequencing and genome-wide array data to evaluate the degree to which bottleneck-enriched protein-altering alleles and unequivocally implicated common variants contribute an excess $\mathrm{CD}$ genetic risk to $\mathrm{AJ}[6]$. Despite the progress in mapping genes and alleles for rare diseases with increased prevalence in the AJ population, precise estimates of the risk-allele frequency and the carrier rate in the AJ population have not yet been determined[8]. Through this study, we provide a frequency resource of protein-coding alleles from over 2,000 non-CD $\mathrm{AJ}$ individuals with low admixture that will serve to improve interpretation of rare disease risk alleles in the AJ population and which we employ to discover new Crohn's risk alleles by comparison to 1855 AJ Crohn's cases.

\section{Results}

We generated a jointly called whole-exome sequence dataset consisting of 18,745 individuals from international Inflammatory Bowel Disease (IBD) and non-IBD cohorts[9,10] (S1 Fig). Given the increased prevalence of Crohn's disease in the AJ population, our global sequencing efforts had specifically included 5,652 individuals self-reporting as Jewish and, as we aimed to focus on variation observed in the AJ population in comparison to reference populations in ExAC[9,11] (including non-Finnish Europeans (NFE), Latino (AMR), and African/AfricanAmerican (AFR)) populations, we chose a model-based approach to estimate the ancestry of the study population using ADMIXTURE[12].

To identify AJ individuals and estimate admixture fractions we used a set $(n=21,066)$ of LD-pruned common variants (MAF $>1 \%$, see Supplementary Note for additional details) filtered for genotype quality $(\mathrm{GQ}>20)$. The 18,745 individuals were assigned to four groups $(\mathrm{K}=4)$ using ADMIXTURE (further described in Supplementary Note, also see S3 Fig). One group of 5,685 individuals was found consisting mostly (84\%) of self-reported AJ individuals, while 3,522 of these individuals were further found with high ancestry fraction $(>0.9)$ mapping to this group (S2 Fig, S1 Table). Thus, many self-reported AJ individuals were not included, as they did not have high enough ascertained $\mathrm{AJ}$ ancestry fraction. As we were interested in computing an enrichment statistic that would not be affected by possible admixture, we obtained alternate (non-reference) allele frequency estimates by restricting the enrichment analysis to the 2,178 non-IBD Ashkenazi Jewish individuals that passed QC and relatedness filtering and had $\mathrm{AJ}$ ancestry fraction (genotype ancestry grouping closely with other $\mathrm{AJ}$ individuals) of $>0.9$. Our study includes exomes throughout Europe and Israel but the vast majority 
(86\%) of these high ancestry fraction AJ individuals were collected in major US cities including Los Angeles, Boston, Baltimore, and New York (S2 Table).

To explore AJ exome population genetics, including proportion of enriched alleles and degree of enrichment, we used the observed alternate allele counts and total number of alleles available from ExAC release 0.3 dataset [ $n_{\text {total }}=60,706$; NFE $(n=31,902$; after excluding AJ individuals from ExAC), AFR $(n=5,203)$, and AMR $(n=5,789)]$. We focused on protein-coding alleles with estimated allele frequency of at least 0.002 and less than .1 in $\mathrm{AJ}\left(\mathrm{n}_{\text {alleles }}=\right.$ 73,228; practical cutoff of what could be statistically defined as convincing enrichment, see S4 Fig), and applied a one-sided Fisher's exact test on allele counts (see Supplementary Note), to classify the observed alleles into two groups: "enriched" or "not enriched". This analysis identified $34 \%$ of protein-coding alleles as significantly enriched, with mean 15 -fold increased odds of the alternate allele compared to other populations. Different proportions of alleles belong to the enriched group depending on variant annotation: $36 \%$ for predicted protein-truncating variants (PTV); $38 \%$ for predicted protein-altering variants (PRA); and 31\% for synonymous variants. The substantially higher PTV+PRA:synonymous ratio observed in the enriched category is consistent with those alleles being drawn randomly from a large pool of much rarer alleles (where the functional:synonymous ratio is higher[3]) and abruptly boosted in frequency (Fig $1, \mathrm{p}<10^{-16}$ across comparisons of PTV and PRA to synonymous variants, two-proportion test, Supplementary Note). Since much rarer alleles have a higher probability of being damaging (e.g., they have a higher missense/synonymous ratio), the advantage to gene mapping arises from the fact that enriched alleles of a certain frequency are more damaging/deleterious on average than non-enriched alleles of the same frequency.

Additionally, we may expect that a "depleted" set of alleles arises from the founder effect, but in reality, many of these already rare variants are simply eliminated during the bottleneck. Of course, it is more difficult and less interesting to search for depleted alleles, as their absence provides no opportunity to obtain significant statistics on population enrichment or disease association.

We intersected the list of protein-coding alleles identified in the AJ exome sequencing study with alleles reported to be pathogenic with no conflicting evidence $(n=42,226)$ in ClinVar[14] resulting in 148 alleles found both in ClinVar and with p-value less than .005 of belonging to the AJ enriched set (S1 Data File). In OMIM, 48 of the 148 alleles included documentation of a disease subject with $\mathrm{AJ}$ ancestry (Table 1). This set of enriched alleles includes all of the major AJ mutations for 8 diseases described in the American College of Medical Genetics and Genomics 2008 screening guideline study[15]. In the setting of autosomal recessive disorders these differences in population allele frequencies may contribute a factor proportional to the squared enrichment difference to genetic risk and prevalence between populations (see Supplementary Note). For instance, a 19-fold enriched frameshift indel, p. Tyr427llefsTer5, in HEXA, contributes a 361-fold enrichment in genetic risk in AJ to non-AJ population to Tay-Sachs disease. Enrichment in this large adult Ashkenazi exome database reinforces recent publications of founder mutations for rare pediatric disorders including FKTN (Walker Warburg syndrome)[16], CCDC65 (Primary ciliary dyskinesia)[17], TMEM216 (Joubert syndrome)[18], C11orf73 (Leukoencephalopathy)[19]; PEX2 (Zellweger syndrome) [20], VPS11 (Hypomyelination and developmental delay)[21] and BBS2 (Bardet-Biedl syndrome)[22]. While many alleles on this pathogenic list may demonstrate incomplete penetrance (as in the case of p.V726A in MEFV[23] for Familial Mediterranean fever) and some may not show recessive inheritance, this resource should provide considerable assistance in gene discovery and clinical genetic screening in AJ (S2 Data File).

To assess whether AJ-enriched protein-coding alleles also contribute to the established difference in CD genetic risk we performed case-control association analyses. Since individuals 
A

Protein-coding alleles enrichment histogram

$$
\square \text { not enriched } \square \text { enriched }
$$

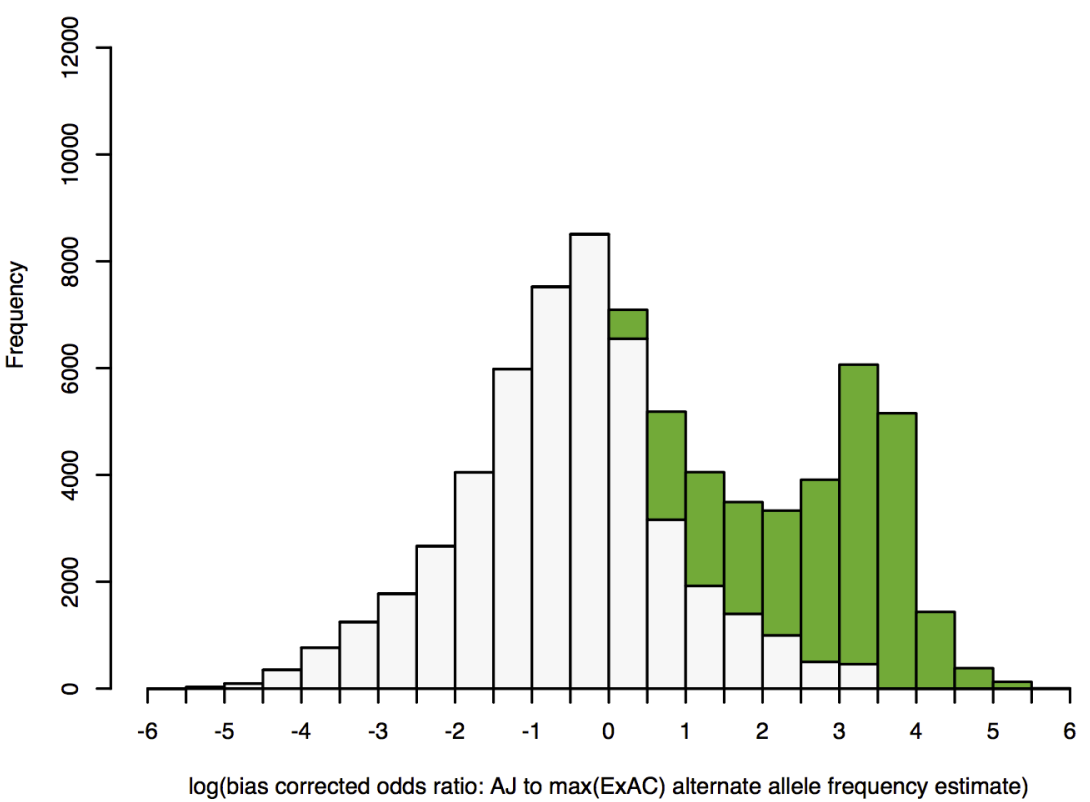

B

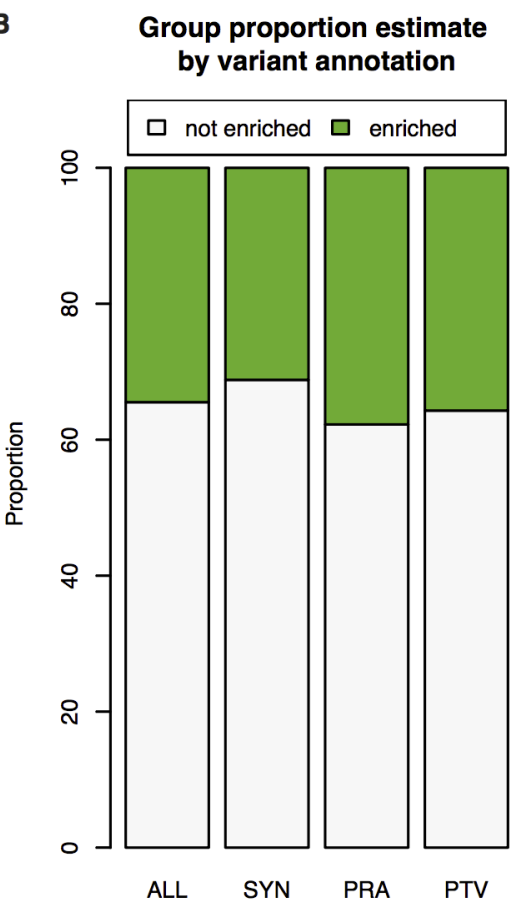

Fig 1. Enrichment of alleles discovered in AJ exome sequencing project. A) Histogram of estimated log enrichment statistic, defined as the log of the bias corrected odds ratio comparing the allele frequency in AJ population to the maximum allele frequency estimated from NFE, AFR, and AMR populations in ExAC. For each histogram bin we show a bar plot of the expected number of alleles belonging to the two groups we analyzed: 1) enriched (green) and 2) not enriched (white). B) Bar plots of estimated percentage of alleles belonging to the two groups we analyzed for all protein-coding (ALL), synonymous (SYN), protein-altering (PRA), and protein-truncating variants (PTV). An estimate of $34 \%$ of protein-coding alleles observed in AJ have a mean shift of 15 -fold increased odds of the alternate allele compared to other reference populations. This observation is supported by the property that compared to intergenic variants, coding variants tend to be younger for a given frequency and the more pathogenic a variant, the younger it is, therefore tending to be population specific[13].

https://doi.org/10.1371/journal.pgen.1007329.g001

with only partial AJ ancestry will still carry bottleneck-enriched alleles, here we included samples with estimated AJ ancestry fraction $>0.4$ (Supplementary Note, S2 Fig), resulting in a dataset of 4,899 AJ samples (1,855 Crohn's disease and 3,044 non-IBD). To improve ability to detect a true association, we performed a meta-analysis with CD and non-IBD case-control exome sequencing data from two additional ancestry groups: 1) non-Finnish European (NFE) (2,296 CD and 2,770 non-IBD); and 2) Finnish (FIN) (210 CD and 9,930 non-IBD samples) from a separate callset described in a previous publication[24] for a total of 4,361 CD samples and 15,744 non-IBD samples. By calling additional non-AJ samples, we hoped to discern which of the AJ-enriched alleles contributed a significant risk factor across all populations. The meta-analysis performed across several populations described should mitigate biases by confirming consistency in effect size across these population groups.

Study-specific association analysis was performed with Firth bias-corrected logistic regression $[25,26]$ and four principal components as covariates using the software package EPACTS [27] (S5 Fig). We combined association statistics in a meta-analysis framework using the Bayesian models in Band et al.[28]. We used the correlated effects model, obtained a Bayes factor $(\mathrm{BF})$ by comparing it with the null model where all the prior weight is on an effect size of zero, reported p-value approximation using the $\mathrm{BF}$ as a test statistic, and assessed whether heterogeneity of effects exist across studies for downstream QC (see Supplementary Note). We 
Table 1. Forty-eight ClinVar "pathogenic" alleles enriched in AJ. HGVS and Gene is the allele nomenclature in ClinVar and gene symbol, respectively. Enrichment odds ratio corresponds to the bias corrected comparison of allele frequency in $\mathrm{AJ}(\mathrm{AJ} \mathrm{AF}$ ) to maximum frequency among three population groups (max EXAC AF): 1) NFE; 2) AMR; and 3) AFR. Curated trait is based on the trait description in the Online Mendelian Inheritance in Man (OMIM) and is independent of effect size as a Crohn's risk allele. Inheritance corresponds to the inheritance description in OMIM (AR: autosomal recessive, AD: autosomal dominant, risk factor: not specified genetic risk factor). Alleles are sorted in decreasing order by AJ AF.

\begin{tabular}{|c|c|c|c|c|c|c|c|}
\hline Variant & HGVS & Gene & $\begin{array}{l}\text { Enrichment Odds } \\
\text { Ratio }\end{array}$ & AJ AF & $\begin{array}{c}\text { Max ExAC } \\
\text { AF }\end{array}$ & Curated Traits & Inheritance \\
\hline 16:3293310:A:G & p.Val726Ala & $M E F V$ & 26.08 & 0.0416 & 0.0017 & Familial Mediterranean fever & $\mathrm{AR}$ \\
\hline 5:150723155:C:A & p.Gly87Val & SLC36A2 & 3.51 & 0.0414 & 0.0122 & Hyperglycinuria & $\mathrm{AD}$ \\
\hline 1:155205634:T:C & p.Asn409Ser & $G B A$ & 11.16 & 0.0296 & 0.0027 & $\begin{array}{l}\text { Susceptibility to Lewy bod dementia, Gaucher's disease, } \\
\text { Susceptibility to late onset Parkinson's disease }\end{array}$ & $\mathrm{AR}$ \\
\hline 4:187201412:T:C & p.Phe301Leu & $F 11$ & 47.17 & 0.0273 & 0.0006 & Hereditary factor XI deficiency & $\mathrm{AR}$ \\
\hline $\begin{array}{l}\text { 13:20763553:CA: } \\
\text { C }\end{array}$ & p.Leu56Argfs & GJB2 & 39.19 & 0.0199 & 0.0005 & Autosomal recessive deafness & $\mathrm{AR}$ \\
\hline 4:187195347:G:T & p.Glu135Ter & $F 11$ & 28.20 & 0.0195 & 0.0007 & Factor XI deficiency & $\mathrm{AR}$ \\
\hline 12:14421038:G:A & p.Arg49Cys & PRB3 & 16.12 & 0.0189 & 0.0012 & Salivary peroxidase & $\mathrm{AR}$ \\
\hline 9:111662096:A:G & c. $2204+6 \mathrm{~T}>\mathrm{C}$ & IKBKAP & 45.22 & 0.0168 & 0.0004 & Familial dysautonomia & $\mathrm{AR}$ \\
\hline $\begin{array}{l}\text { 15:72638920:G: } \\
\text { GGATA }\end{array}$ & p.Tyr427IlefsTer5 & HEXA & 19.14 & 0.0122 & 0.00064 & Tay-Sachs disease & $\mathrm{AR}$ \\
\hline 1:125848678:C:T & p.Arg4192His & USH $2 \mathrm{~A}$ & 13.63 & 0.0106 & 0.0008 & Retinitis pigmentosa & $\mathrm{AR}$ \\
\hline 22:29091207:G:A & p.Ser428Phe & CHEX2 & 50.06 & 0.0103 & 0.0002 & Hereditary cancer, multiple types & Risk factor \\
\hline $\begin{array}{l}\text { 10:99371368: } \\
\text { TGAG:T }\end{array}$ & p.Glu315del & HOGA1 & 29.28 & 0.0101 & 0.0003 & Primary hyperoxaluria & AR \\
\hline 7:117282620:G:A & p.Trp1282Ter & CFTR & 23.64 & 0.0085 & 0.0004 & Cystic fibrosis & $\mathrm{AR}$ \\
\hline 11:17418602:C:T & c.3992-9G $>A$ & $A B C C 8$ & 40.62 & 0.0076 & 0.0002 & Hyperinsulinemic hypoglycemia & $\mathrm{AR}, \mathrm{AD}$ \\
\hline 17:3402294:A:C & p.Glu285Ala & $A S P A$ & 40.36 & 0.0076 & 0.0002 & Canavan disease & $\mathrm{AR}$ \\
\hline 2:98986540:G:A & c. $101+1 \mathrm{G}>\mathrm{A}$ & CNGA3 & 26.11 & 0.0074 & 0.0003 & Achromatopsia & $\mathrm{AR}$ \\
\hline $\begin{array}{l}\text { 13:32914437:GT: } \\
\text { G }\end{array}$ & p.Ser1982Argfs & $B R C A 2$ & 27.57 & 0.0069 & 0.0003 & Hereditary cancer, multiple types & Risk factor \\
\hline 9:97934315:T:A & c. $456+4 \mathrm{~A}>\mathrm{T}$ & FANCC & 42.75 & 0.0069 & 0.0002 & Fanconi anemia & $\mathrm{AR}$ \\
\hline $\begin{array}{l}\text { 9:108382330:G: } \\
\text { GA }\end{array}$ & p.Phe390Ilefs & FKTN & 32.62 & 0.0067 & 0.0002 & Limb-girdle muscular dystrophy-dystroglycanopathy & $\mathrm{AR}$ \\
\hline 12:40734202:G:A & p.Gly2019Ser & LRRK2 & 20.64 & 0.0064 & 0.0003 & Parkinson's disease & Risk factor \\
\hline 17:41055964:C:T & p.Arg83Cys & G6PC & 11.04 & 0.0062 & 0.0006 & Glycogen storage disease & $\mathrm{AR}$ \\
\hline 1:26764719:A:G & p.Lys42Glu & DHDDS & 64.83 & 0.0051 & 0.0001 & Retinitis pigmentosa & $\mathrm{AR}$ \\
\hline 3:150690352:A:C & p.Asn48Lys & CLRN1 & 46.26 & 0.0051 & 0.0001 & Usher syndrome & $\mathrm{AR}$ \\
\hline $\begin{array}{l}\text { 12:49312533: } \\
\text { GTA:G }\end{array}$ & p.Ile293Profs & CCDC65 & 25.75 & 0.0048 & 0.0002 & Ciliary dyskinesia without situs inversus & $\mathrm{AR}$ \\
\hline 6:80878662:G:C & p.Arg183Pro & $B C K D H B$ & 29.42 & 0.0046 & 0.0002 & Maple syrup disease & $\mathrm{AR}$ \\
\hline 10:56077147:G:A & p.Arg245Ter & PCDH15 & 26.58 & 0.0046 & 0.0002 & Usher syndrome & $\mathrm{AR}$ \\
\hline 7:107555951:G:T & p.Gly229Cys & $D L D$ & 26.55 & 0.0046 & 0.0002 & Maple syrup disease & $\mathrm{AR}$ \\
\hline 15:72638575:C:G & c. $1421+1 \mathrm{G}>\mathrm{C}$ & HEXA & 52.65 & 0.0044 & 0.0001 & Tay-Sachs disease & $\mathrm{AR}$ \\
\hline 15:72105913:G:A & p.Arg311Gln & NR2E3 & 9.86 & 0.0042 & 0.0004 & Enhanced s-cone syndrome & $\mathrm{AR}$ \\
\hline 5:178699927:G:A & p.Gln225Ter & ADAMTS2 & 129.41 & 0.0041 & 0.0000 & Ehlers-Danlos syndrome, dermatosparaxis type & $\mathrm{AR}$ \\
\hline 16:50745656:G:A & p.Ala612Thr & NOD2 & 12.48 & 0.0039 & 0.0003 & Early-onset sarcoidosis & Risk factor \\
\hline 11:6415434:G:T & p.Arg498Leu & SMPD1 & 41.53 & 0.0039 & 0.0001 & Niemann-Pick disease & $\mathrm{AR}$ \\
\hline 11:61161437:G:T & p.Arg73Leu & THEM 216 & 27.77 & 0.0039 & 0.0001 & Joubert syndrome & AR \\
\hline $\begin{array}{l}\text { 1:53676583:CAG: } \\
\text { C }\end{array}$ & pLys414ThrfsTer7 & CPT2 & 78.34 & 0.0037 & 0.0000 & Carnitine palmitoyltransferase II deficiency & $\mathrm{AR}$ \\
\hline 1:53676688:T:C & p.Phe448Leu & CPT2 & 78.35 & 0.0037 & 0.0000 & Carnitine palmitoyltransferase II deficiency & $\mathrm{AR}$ \\
\hline 3:172737276:C:T & p.Arg283Gln & SPATA16 & 9.79 & 0.0037 & 0.0004 & Spermatogenic failure & $\mathrm{AR}$ \\
\hline 11:86017416:G:C & p.Val54Leu & C11orf73 & 47.03 & 0.0037 & 0.0001 & Hypomyelinating leukodystrophy & AR \\
\hline
\end{tabular}


Table 1. (Continued)

\begin{tabular}{|c|c|c|c|c|c|c|c|}
\hline Variant & HGVS & Gene & $\begin{array}{l}\text { Enrichment Odds } \\
\text { Ratio }\end{array}$ & AJ AF & $\begin{array}{c}\operatorname{Max} \text { ExAC } \\
\text { AF }\end{array}$ & Curated Traits & Inheritance \\
\hline 8:77896070:G:A & p.Arg119Ter & PEX2 & 20.03 & 0.0034 & 0.0002 & Peroxisome biogenesis disorder & $\mathrm{AR}$ \\
\hline 11:118951899:T:G & p.Cys845Gly & VPS11 & 190.98 & 0.0030 & 0.0000 & Hypomyelinating leukodystrophy & $\mathrm{AR}$ \\
\hline 6:80203353:G:A & p.Gln279Ter & $L C A 5$ & 29.25 & 0.0028 & 0.0001 & Leber congenital amaurosis & $\mathrm{AR}$ \\
\hline 19:7591645:A:G & c. $406-2 \mathrm{~A}>\mathrm{G}$ & MCOLN1 & 21.93 & 0.0028 & 0.0001 & Mucolipidosis & $\mathrm{AR}$ \\
\hline 16:56530894:C:G & p.Arg632Pro & BBS2 & 29.37 & 0.0028 & 0.0001 & Retinitis pigmentosa & $\mathrm{AR}$ \\
\hline $\begin{array}{l}\text { 17:41276044: } \\
\text { ACT:A }\end{array}$ & p.Glu23Valfs & $B R C A 1$ & 10.04 & 0.0025 & 0.0003 & Hereditary cancer, multiple types & Risk factor \\
\hline 4:100543913:G:T & p.Gly865Ter & MTTP & 40.38 & 0.0025 & 0.0001 & Abetalipoproteinaemia & AR \\
\hline 2:99013302:G:A & p.Gly557Arg & CNGA3 & 29.36 & 0.0023 & 0.0001 & Achromatopsia & $\mathrm{AR}$ \\
\hline 7:107557794:G:A & p.Glu375Lys & $D L D$ & 26.44 & 0.0021 & 0.0001 & Maple syrup disease & $\mathrm{AR}$ \\
\hline 17:41209079:T:TG & p.Gln1756Profs & $B R C A 1$ & 8.80 & 0.0021 & 0.0002 & Hereditary cancer, multiple types & Risk factor \\
\hline 10:99371292:G:T & p.Gly287Val & HOGA1 & 22.01 & 0.0021 & 0.0001 & Primary hyperoxaluria & AR \\
\hline
\end{tabular}

https://doi.org/10.1371/journal.pgen.1007329.t001

separately assessed CD associations of enriched protein-altering (PRA) and synonymous $(\mathrm{SYN})$ alleles in protein-coding genes in CD implicated GWAS loci $\left(\mathrm{n}_{\mathrm{gwas}, \text { pra }}=351 ; \mathrm{n}_{\mathrm{gwas}, \mathrm{syn}}=\right.$ 167 ), and outside implicated GWAS loci ( $\mathrm{n}_{\text {non-gwas,pra }}=12,529 ; \mathrm{n}_{\text {non-gwas,syn }}=6,202$, Fig 2$)$. See Methods and Materials for a description of these loci.

We identified ten $\mathrm{AJ}$ enriched $\mathrm{CD}$ risk alleles $(\mathrm{p}<0.005)$ : the previously published risk haplotypes in LRRK2 and NOD2 (LRRK2: p.N2081D; NOD2: p.N852S, p.G908R, p.M863V+p. fs1007insC)[29,30], in addition to newly implicated alleles (NOD2: p.A612T, $\mathrm{p}=2.8 \times 10^{-9} ; \mathrm{c} .74-$ $7 \mathrm{~T}>\mathrm{A}, \mathrm{p}=1.4 \times 10^{-4} ; \mathrm{p} . \mathrm{L} 248 \mathrm{R}, \mathrm{p}=6.4 \times 10^{-4} ; \mathrm{p} . D 357 \mathrm{~A}, \mathrm{p}=0.0011 ;$ LRRK2: $\mathrm{p} . \mathrm{G} 2019 \mathrm{~S}, \mathrm{p}=0.0014$, a Parkinson's disease risk allele[31]). To assess whether the new NOD2 enriched alleles are conditionally independent of the previously established associated NOD2 alleles we performed conditional haplotype association analysis in PLINK and Bayesian model averaging[32] for variable selection, both of which suggested independent effects for all alleles (S6 Fig, S3 Table).

Deviation from additivity can contribute additionally to individual risk but has been difficult to document in complex disease associations with modest ORs. Despite the functional relationship between $L R R K 2$ and $N O D 2$ [33], we do not observe deviation from additivity between LRRK2 and NOD2 ( $\mathrm{p}=0.273$ ); that is, the effect of mutations in both LRRK2 and NOD2 is no greater than the sum of their individual effects. We assessed whether composite risk carriers (carrier of more than one variant allele) had evidence of deviation from additivity. Deviation from additivity has been reported for p.fs1007insC, p.G908R, and p.R702W in NOD2[34,35]. In our AJ exome sequencing data set we estimate a 1-hit effect equal to 1.82 (95\% confidence interval $[1.59,2.07])$ and a 2 -hit effect equal to 8.24 (95\% confidence interval $[6.06,11.21]$; we found similar evidence for departure from additivity when restricting the analysis to the newly reported alleles only: $\mathrm{p}=0.00357$, odds ratio $=7.53$ ). We confirmed this finding using the larger non-AJ Crohn's disease ImmunoChip dataset to provide a more precise estimate of the 1-hit effect $(\mathrm{OR}=2.17 ; 95 \%$ confidence interval [2.07, 2.27], S4 Table) and the non-additive 2 -hit effects in NOD2 (OR $=9.93$; 95\% confidence interval [8.88, 11.13], S5 Table). We found no evidence of deviation from additivity for the associated protein-altering alleles in LRRK2 ( $\mathrm{p}=0.418)$.

Given that enriched genetic variants in NOD2 and LRRK2 contribute to differences in CD risk in AJ population, we next asked whether unequivocally established common variant associations contribute to differences in CD genetic risk. We performed polygenic risk score (PRS) analysis using reported effect size estimates from $124 \mathrm{CD}$ alleles including those reported in a 
A

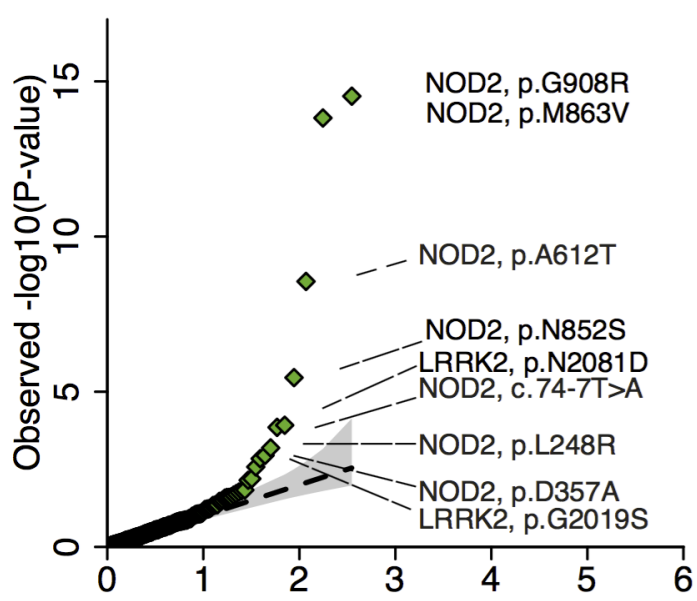

C

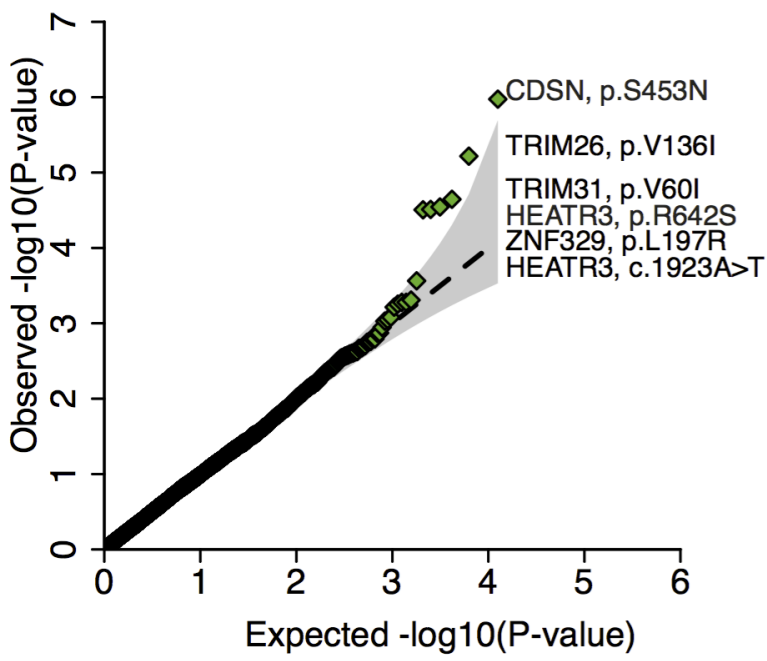

B

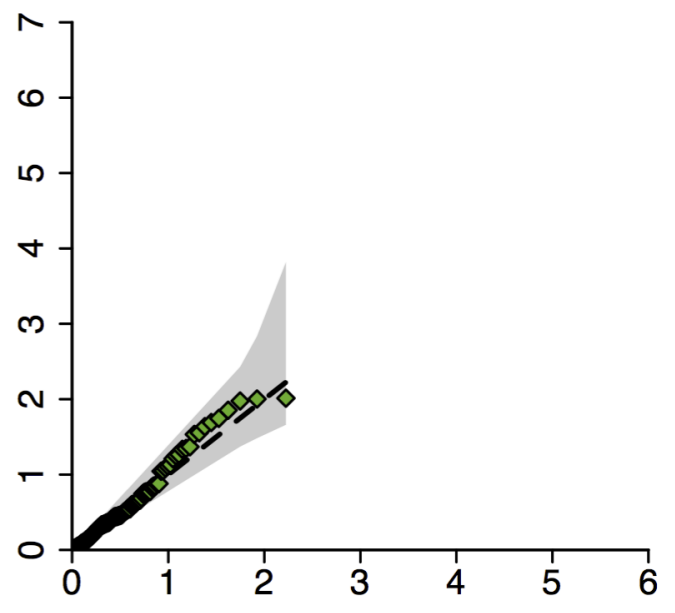

D

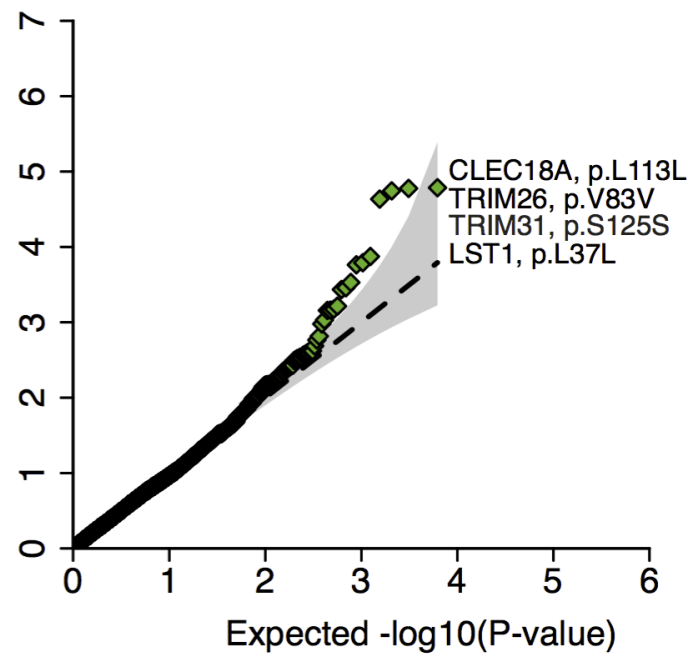

Fig 2. Q-Q plots of enriched alleles. Q-Q plots of Crohn's disease association for AJ enriched A) protein-altering (protein-truncating and missense) and B) synonymous alleles in GWAS regions; and AJ enriched C) protein-altering and D) synonymous alleles outside of GWAS regions. For each $\mathrm{Q}-\mathrm{Q}$ plot variants with a corresponding $\mathrm{p}$-value less than or equal to a threshold where expected number of false discoveries is equal to one are annotated. The black dashed line is $y=x$, and the grey shapes show $95 \%$ confidence interval under the null.

https://doi.org/10.1371/journal.pgen.1007329.g002

previously published study [36] and four variants in IL23R from a recent fine-mapping study [37], and excluding variants in NOD2 and LRRK2. We observed an elevated PRS for AJ compared to non-Jewish controls ( 0.97 s.d. higher, $\mathrm{p}<10^{-16}$; Fig $3 \mathrm{~A}$; number of non-AJ controls = 35,007; number of AJ controls = 454), and as expected when performing the PRS analysis using OR calculated from non-Jewish subset of iCHIP data the signal still remains $\left(\mathrm{p}<10^{-16}\right.$, S7 Fig). We observed a similar trend for the CD samples ( 0.54 s.d. higher; $\mathrm{p}<10^{-16}$; Fig 3B; number of non-AJ CD cases $=20,652$; number of AJ CD cases $=1,938$ ). We demonstrate this is not a systematic property of common risk alleles in AJ by running the same comparison using instead the comparable set of established schizophrenia associated alleles from the Psychiatric Genomics Consortium[38]. 
A

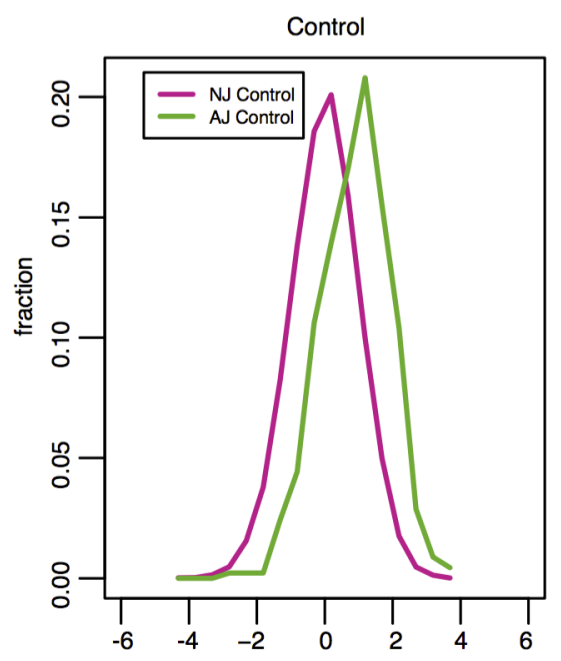

B

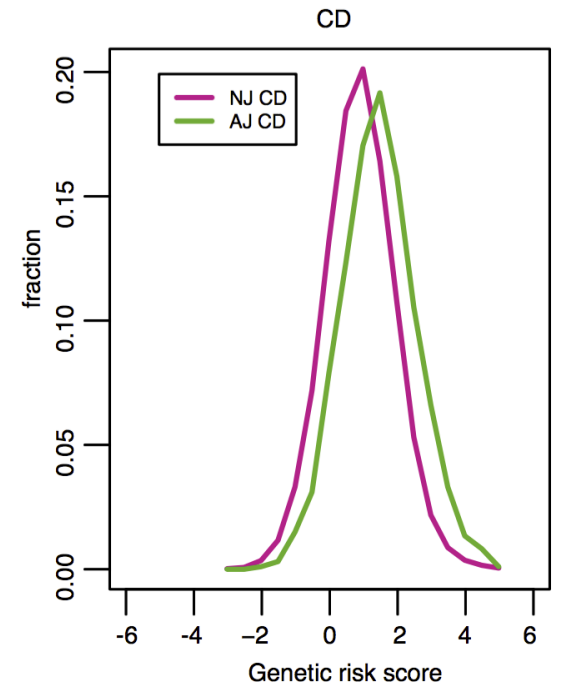

C
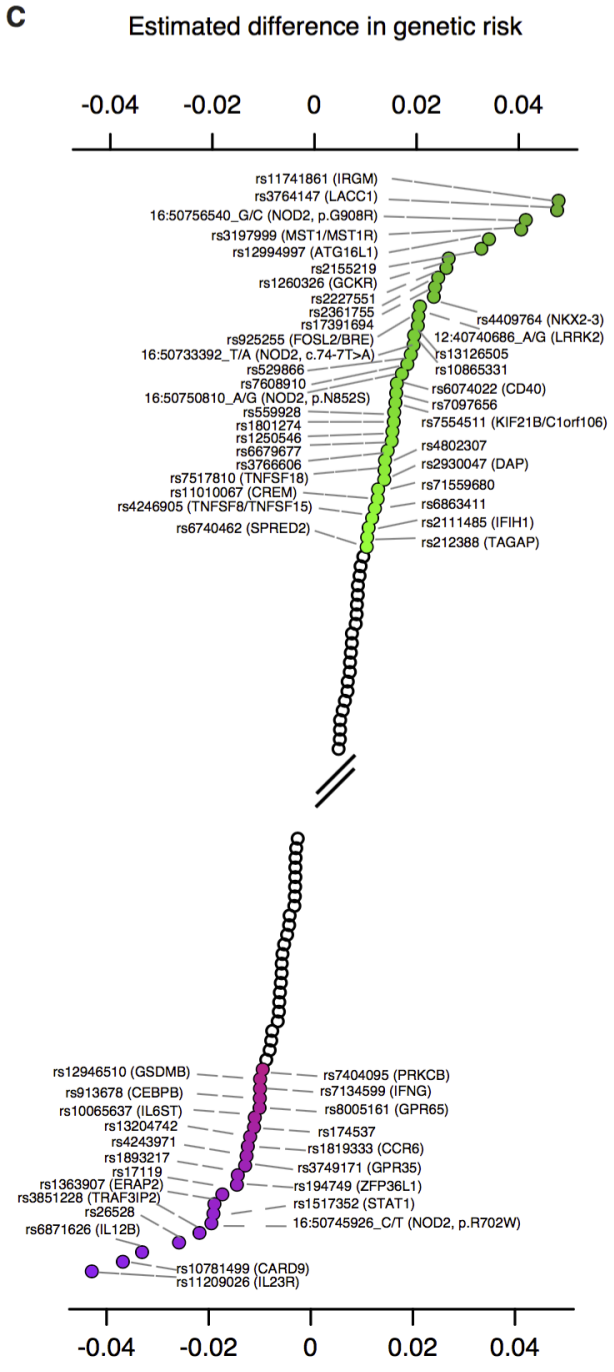

Fig 3. AJ individuals have higher CD polygenic risk score than NJ controls. NJ: non-Jewish; AJ: Ashkenazi Jewish; CD: Crohn's disease; PRS: polygenic risk score. A) Density plot of CD polygenic risk scores in $454 \mathrm{AJ}$ (green) and 35,007 NJ(purple)controls. AJ controls have higher CD polygenic risk score than NJ controls $\left(0.97\right.$ s.d. higher, $\left.\mathrm{p}<10^{-16}\right)$. B) Density plot of CD polygenic risk scores in $1,938 \mathrm{AJ}$ (green) and 20,652 NJ CD (purple) cases $\left(0.54\right.$ s.d. higher, $\mathrm{p}<10^{-16}$ ). For both density plots the scores have been scaled to NJ controls, thus resulting in an NJ control PRS density of mean equal to 0 and variance equal to 1 (see Online Methods). C) Ranked (decreasing order) $\mathrm{CD}$ associated variants by estimated contribution to the differences in genetic risk between $\mathrm{AJ}$ and NJ. Associated variants with estimated contribution greater than or equal to 0.01 , computed as $2 \log$ (odds ratio) (AJ frequency-NJ frequency), assuming additive effects on the log scale, are highlighted in green. Associated variants with estimated contribution less than or equal to -0.01 are highlighted in purple. Forward slashes represent a break in variants highlighted.

https://doi.org/10.1371/journal.pgen.1007329.g003

To quantify the relative contribution of $\mathrm{CD}$-implicated alleles to the difference in genetic risk between $\mathrm{AJ}$ and non-AJ populations we estimated the expected PRS value of an individual and expected difference in PRS between two populations by simply using summary statistics including the frequency of the minor allele in the two populations and the corresponding odds ratio (Supplementary note, S6 Fig).

We applied the approach to all CD implicated alleles and observed that variants in GWAS loci annotated as IRGM, LACC1, NOD2, MST1, ATG16L1, GCKR, NKX2-3, and LRRK2[36] contribute substantially $(>0.01)$ to the increased genetic risk observed in AJ. It is possibly 
relevant that variants contributing to increased risk in AJ include many autophagy/intracellular defense genes (IRGM, ATG16L1, LRRK2), while those contributing to increased risk in non-AJ include many anti-fungal/Th17/ILC3 genes[39] (IL23R, IL12B, CARD9, TRAF3IP2, IL6ST, CEBPB; Fig 3C).

Both documented variability in the occurrence of CD over time[40,41] and substantial uncertainty in reported $\mathrm{CD}$ prevalence estimates $[42,43]$ impact our ability to precisely estimate the overall contribution of genetics to the established difference in prevalence between populations. To interpret the impact of shifts in genetic risk score on differences in prevalence, we used the logit risk model[35] and evaluated a new estimate of disease probability, $\mathrm{p}_{\text {new }}$, assuming an initial disease probability, $\mathrm{p}_{0}$, and multiple values for the differences in genetic risk.

Assuming log-additive effects, and a logit-risk model, we estimate that the observed differences in genetic risk between the $\mathrm{AJ}$ and non-AJ populations contribute an expected 1.5-fold increase in disease prevalence in a population with environmental risk factors corresponding to $\mathrm{AJ}$ and baseline genetic risk corresponding to non-AJ populations (S7-S9 Figs). To address the extent to which non-additive effects in NOD2 may impact the observed prevalence we assumed 1-hit and 2-hit odds ratios of 2.17 and 9.93 , respectively. We attribute a $6.8 \%$ difference in the ratio of estimated disease prevalence in the AJ population to the deviation from additivity, suggesting a small effect on differences in population prevalence (Supplementary Note).

\section{Discussion}

Analyzing data from 5,685 Ashkenazi Jewish exomes, we provide a systematic analysis of AJ enriched protein-coding alleles, which may contribute to differences in genetic risk to $\mathrm{CD}$ as well as numerous rare diseases, many of which are transmitted via autosomal recessive inheritance. We identified protein-altering alleles in NOD2 and LRRK2 that are conditionally independent and contribute to the excess burden of $\mathrm{CD}$ in AJ. We found evidence that common variant risk defined by GWAS shows a strong elevated difference between $\mathrm{AJ}$ and non-AJ European population samples ( 0.97 s.d. higher in controls, 0.54 s.d. higher in cases, $\mathrm{p}<10^{-16}$ in both), independent of NOD2 and LRRK2[44]. Highly polygenic diseases are unlikely to have substantially altered incidence as a result of a bottleneck alone-for every enriched variant there are those depleted or lost entirely and population genetics simulations[45] suggest no systematic alteration of overall genetic burden as a function of a bottleneck. Thus, the strong (approximately 1.5-fold, see supplementary note) difference in Crohn's incidence in concert with a systematic enrichment of risk-increasing alleles, unlikely to have arisen by chance, suggests non-random selection in the $\mathrm{AJ}$ population for higher $\mathrm{CD}$ risk alleles. It seems plausible that, rather than 'selection for Crohn's' per se, this likely suggests a subset of Crohn's risk alleles may contribute to a common biological process (e.g., a specific immune response) or phenotype that was positively selected for in AJ[46-48]. Such weak, widespread 'polygenic selection' has previously been observed with respect to height-associated SNPs in Europe[49], where drift alone could not explain the systematic enrichment of height-increasing alleles in populations of Northern Europe vs. Southern Europe. We found that CD risk alleles that are systematically elevated in AJ are not unusually elevated in another well-established founder population for which we have extensive genotype data (Finland). In Finns, Crohn's risk alleles were not systematically enriched-they were if anything slightly depleted with 69 risk alleles at higher frequency in Finns than NFE and 79 risk alleles at lower frequency in Finns than NFE. We also demonstrate this is not a systematic property of common risk alleles in AJ by running the same comparison using instead the comparable set of established schizophrenia associated alleles from the Psychiatric Genetics Consortium[50]. We mapped 102 schizophrenia- 
associated index SNPs to AJ frequency data and again observed no uneven distribution where risk alleles are systematically more or less common. In total, 52 risk alleles were at higher frequency in AJ than NFE and 50 risk alleles were higher frequency in NFE than AJ.

This study of $\mathrm{CD}$ in the AJ population confirms population-genetic expectations. First, recently bottlenecked populations are uniquely powered to discover alleles with markedly increases in frequency, and, as a consequence, contributors to differences in genetic risk across population groups. Second, while NOD2 and published common variant associations contribute substantially to the genetic risk of $\mathrm{CD}$, other genes with causal alleles that failed to pass through the bottleneck are missed, consistent with predictions from Zuk et al[4].

We provide an exome frequency resource of protein-coding alleles in AJ along with estimates of population-specific enrichment. The sets of enriched alleles should be carefully considered when performing case-control analysis. Population structure can easily lead to false positive associations, especially for low frequency and rare variants, if the AJ:nonAJ ratio is slightly different in cases and controls. Our approach and this resource will likely catalyze our understanding of the medical relevance of enriched alleles in population isolates. Most importantly, the frequency reference provides critical guidance in pinpointing or excluding specific risk factors in individuals in clinical and research settings.

\section{Materials and methods}

\section{Initial variant call set}

We generated a jointly called dataset consisting of 18,745 individuals from international IBD and non-IBD cohorts. Sequencing of these samples was done at Broad Institute.

\section{Ethics statement}

All patients and control subjects provided informed consent. Recruitment protocols and consent forms were approved by Institutional Review Boards at each participating institutions (Protocol Title: The Broad Institute Study of Inflammatory Bowel Disease Genetics; Protocol Number: 2013P002634). All DNA samples and data in this study were denominalized.

\section{Cohort descriptions}

For all cohorts, CD was diagnosed according to accepted clinical, endoscopic, radiological and histological findings.

\section{Target selection}

G4L WES is a project specific product. It combines the Human WES (Standard Coverage) product with an Infinium Genome-Wide Association Study (GWAS) array. In addition to the array adding to the genomics data, it also acts as a concordance QC, linking 14 SNPs to the exome data. The processing of the exome includes Sample prep (Illumina Nextera), hybrid capture (Illumina Rapid Capture Enrichment - 37Mb target), sequencing (Illumina, HiSeq machines, 150bp paired reads), Identification QC check, and data storage (5 years). Our hybrid selection libraries typically meet or exceed $85 \%$ of targets at $20 \mathrm{x}$, comparable to $\sim 60 \mathrm{x}$ mean coverage. The array consists of a 24-sample Infinium array with $~ 245,000$ fixed genome-wide markers, designed by the Broad. On average our genotyping call rates typically exceed $98 \%$.

\section{Pre-processing}

The sequence reads are first mapped using BWA MEM[51] to the GRCh37 reference to produce a file in SAM/BAM format sorted by coordinate. Duplicate reads are marked-these reads 
are not informative and are not used as additional evidence for or against a putative variant. Next, local realignment is performed around indels. This identifies the most consistent placement of the reads relative to potential indels in order to clean up artifacts introduced in the original mapping step. Finally, base quality scores are recalibrated in order to produce more accurate per-base estimates of error emitted by the sequencing machines.

\section{Variant discovery}

Once the data has been pre-processed as described above, it is put through the variant discovery process, i.e. the identification of sites where the data displays variation relative to the reference genome, and calculation of genotypes for each sample at that site. The variant discovery process is decomposed into separate steps: variant calling (performed per-sample), joint genotyping (performed per-cohort) and variant filtering (also performed per-cohort). The first two steps are designed to maximize sensitivity, while the filtering step aims to deliver a level of specificity that can be customized for each project.

Variant calling is done by running Genome Analysis Toolkit's (GATK) HaplotypeCaller in gVCF mode on each sample's BAM file(s) to create single-sample gVCFs. If there are more than a few hundred samples, batches of $\sim 200 \mathrm{gVCFs}$ are merged hierarchically into a single gVCF to make the next step more tractable. Joint genotyping is then performed on the gVCFs of all available samples together in order to create a set of raw SNP and indel calls. Finally, variant recalibration is performed in order to assign a well-calibrated probability to each variant call in a raw call set, and to apply filters that produce a subset of calls with the desired balance of specificity and sensitivity as described in Rivas et al. (2016)[24]. Samples with $>=10 \%$ contamination are excluded from call sets. Exome samples with less than $40 \%$ of targets at 20X coverage are excluded.

\section{Variant annotation}

Variant annotation was performed using the Variant Effect Predictor (VEP) [cite PMID: 20562413] version 83 with Gencode v19 on GRCh37. Loss-of-function (LoF) variants were annotated using LOFTEE (Loss-Of-Function Transcript Effect Estimator, available at https://github. com/konradjk/loftee), a plugin to VEP. LOFTEE considers all stop-gained, splice-disrupting, and frameshift variants, and filters out many known false-positive modes, such as variants near the end of transcripts and in non-canonical splice sites, as described in the code documentation.

\section{Identification of Finnish samples}

Finnish CD patients were recruited from Helsinki University Hospital and described in more detail previously[52,53]. We used the same exome sequencing dataset described in Rivas et al. [24]. We applied additional PC correction in the Finnish identified individuals to remove individuals with membership of Finnish sub-isolate (Northern Finland) and excluded based on PC2 0.015 (853 excluded, 826 controls, 27 IBD). We recalculated PCs and included the first four PCs in the association analysis.

\section{Identifying previously implicated GWAS loci}

CD implicated GWAS loci were those loci defined as reaching genome-wide significance in International IBD Genetics Consortium studies (Jostins, Ripke et al., Nature 2012) and (Liu et al., Nature Genetics 2015) - Credible sets of SNPs around index associations were defined as in (Huang et al., Nature 2017) for fine-mapped loci, and for others credible sets were defined as all SNPs with $\mathrm{r}^{2}>0.6$ to the index variant. Genes within $50 \mathrm{~kb}$ of the span of credible set SNPs were considered “implicated' by GWAS. 


\section{Ancestry estimation and quality control}

As the present study aimed to focus on variation observed in Ashkenazi Jewish (AJ) population in comparison to reference populations in ExAC including (non-Finnish Europeans (NFE), Latino (AMR), and African/African-American (AFR)) we chose a model-based approach to estimate the ancestry of the study population using ADMIXTURE[12]. To identify AJ individuals and estimate admixture proportions we included a set $(n=21,066)$ of LD-pruned common variants (MAF>1\%) after filtering for genotype quality (GQ>20) using the PLINK LDpruning algorithm, whose description is available at http://pngu.mgh.harvard.edu/ purcell/ plink/summary.shtml\#prune.

For the parameters, we selected a window size of 50 SNPs, a window shift of 5 SNPs at each step, and the variance inflation factor (VIF) threshold equal to 2.

The 18,745 samples were assigned to four groups $(\mathrm{K}=4)$, as ancestry was defined as having a single estimated ancestry fraction $\geq 0.4$, and remaining three fractions $<0.4$ (S2 Fig). Individuals mostly representing African/African-American and East-Asian ancestry (1,267 and 569 individuals respectively) were discarded from downstream analysis, as well as the 983 admixed individuals with none of the ancestry fractions $\geq 0.4$. Thus, a total of 6,093 individuals were considered of Ashkenazi Jewish (AJ) ancestry, while 9,833 were considered to represent Non-Finnish Europeans (NFE). After sample QC and relatedness check, 5,685 individuals of Ashkenazi Jewish and 7,240 of non-Finnish European ancestry were found with valid IBD case/control status (S1 Table). Individuals with Ulcerative Colitis and unspecified and Indeterminate Colitis were further excluded, resulting in 4,899 AJ and 5,066 NFE individuals.

Prior to enrichment and association analysis, 81 samples (of total 18,745) were also filtered due to possible contamination (heterozygous/homozygous ratio $<1$ ), excess of singletons $(\mathrm{n}>2000)$, deletion/insertion ratio $(>1.5)$ and mean genotype quality $(<40) .275$ samples were excluded for relatedness ( $>0.35$ cut-off). Genotypes with low genotype quality $(<20)$ were filtered, in addition to variants with low call rate $(<80 \%)$ and allele balance deviating from 70:30 ratio for greater than $40 \%$ of heterozygous samples if at least 7 heterozygous samples were identified.

As we were interested in computing an enrichment statistic that would not be affected by possible admixture, we obtained alternate allele frequency estimates by restricting the enrichment analysis to the 2,178 non-IBD Ashkenazi Jewish samples that passed QC and relatedness filtering and had AJ focused ancestry fraction $>0.9$ (S1 Fig). Principal Component Analysis (PCA) was done in each ancestry group using the 21,066 variants. Sample QC was done using the Hail software while PCA, differential missingness and sample relatedness analysis was done using PLINK[54]. Hail is an open-source software framework for scalably and flexibly analyzing large-scale genetic data sets (https://github.com/broadinstitute/hail). Allele balance was calculated using PLINK/SEQ (https://atgu.mgh.harvard.edu/plinkseq/).

\section{Estimating fold-enrichment in $\mathrm{AJ}$ population compared to reference populations in ExAC}

Statistical methods: Fisher's exact test. To estimate which alleles are enriched in AJ compared to alleles in reference population groups in ExAC we applied Fisher's exact test onesided alternative ("greater").

Using the number of alternate and reference alleles observed in AJ non-IBD samples and in the population (NFE, AFR or AMR) with the highest frequency from ExAC we compute a bias corrected $\log$ odds ratio estimate, $\widehat{\beta}_{i}$, and its standard error, $\widehat{S E}_{i}$, for odds of the alternate allele as described in the Software DataPlot developed by the National Institute of Standards and Technology (http://www.itl.nist.gov/div898/software/dataplot/refman2/auxillar/logoddra.htm, 
and http://www.itl.nist.gov/div898/software/dataplot/refman2/auxillar/logodrse.htm)

$$
\begin{gathered}
\widehat{\beta}_{i}=\log \left(O R_{i}\right)=\log \left(\frac{\left[\left(0.5+A L T_{A J}\right) \cdot\left(0.5+R E F_{E x A C}\right)\right]}{\left[\left(0.5+R E F_{A J}\right) \cdot\left(0.5+A L T_{E x A C}\right)\right]}\right) \text {, and } \\
\widehat{S E}_{i}^{2}=\frac{1}{0.5+R E F_{A J}}+\frac{1}{0.5+R E F_{E x A C}}+\frac{1}{0.5+A L T_{A J}}+\frac{1}{0.5+A L T_{E x A C}} .
\end{gathered}
$$

Precisely, $\widehat{\beta}_{i}$ is the estimate of the log of the odds ratio of finding the alternate allele in $\mathrm{AJ}$ vs in the ExAC population with the highest allele frequency.

We classified a variant as 'enriched' if p-value was less than .05/73,228, where 73,228 is the number of variants analyzed with minor allele frequency between .002 and .1 .

To estimate allele enrichment in AJ compared to reference populations we used 2,178 nonIBD Ashkenazi Jewish samples, after sample and relatedness QC.

We calculated alternate allele frequencies for the Ashkenazi Jewish population and used allele frequency information for NFE ( $n=31,902$; after excluding AJ individuals from ExAC), $\operatorname{AFR}(n=5,203)$, and $\operatorname{AMR}(n=5,789)$ available from ExAC release 0.3 dataset $\left(n_{\text {total }}=60,706\right)$ and focused on alleles where allele frequency information was available for $\mathrm{AJ}$ and the reference populations. For the enrichment plot we focused on alleles with estimated frequency of at least 0.002 in $\mathrm{AJ}\left(\mathrm{n}_{\text {alleles }}=106,377\right)$ and with alleles observed with an estimated frequency of at least .0001 in the reference populations with depth of coverage of at least $20 \mathrm{X}$ in at least $80 \%$ of the samples in ExAC.

Overlap of enriched alleles with ClinVar. We harmonized the XML and TXT releases of the ClinVar database (April 11, 2016 data release)[14] into a single tab-delimited text file using scripts that we have released publicly (https://github.com/macarthur-lab/clinvar). Briefly, we normalized variants using a Python implementation of vt normalize[55] and de-duplicated to yield a dataset unique on chromosome, position, reference, and alternate allele. A variant was considered 'pathogenic' if it had at least one assertion of either Pathogenic or Likely Pathogenic for any phenotype. A variant was considered 'conflicted' if it had at least one assertion of Pathogenic or Likely Pathogenic, and at least one assertion of Benign or Likely Benign, each for any phenotype. By these criteria, ClinVar contained $n=42,226$ identified as pathogenic and non-conflicted. Intersecting with our dataset revealed that 148 belonged to the $\mathrm{AJ}$ enriched group with p-value less than .005 .

Assessing Crohn's disease association of protein-coding variation that may contribute to difference in disease prevalence in AJ. We focused Crohn's disease association analysis of protein-coding variant to alleles that may account for difference in disease prevalence in AJ population to reference populations. To do so we focused on alleles with high probability of belonging to the enriched group. We included all samples with ADMIXTURE estimated AJ ancestry fraction of at least 0.4 (we excluded any samples that had alternative ancestry fraction of at least 4 in any other group). Samples with Ulcerative Colitis $(n=700)$, unspecified and Indeterminate Colitis $(\mathrm{n}=86)$ were excluded from subsequent analysis. This resulted in a dataset of 4,899 AJ samples (1,855 Crohn's disease and 3,044 non-IBD).

Study-specific association analysis was performed with Firth bias-corrected logistic regression test $[25,26]$ and four principal components as covariates using the software package EPACTS version 3.2.6[27]. Minimum minor allele count $(\geq 1)$ and variant call rate $(\geq 0.8)$ filters were used.

For meta-analysis we combined association statistics using the Bayesian models and frequentist properties proposed in Band et al[28], which is a normal approximation to the logistic regression likelihood suggested by Wakefield[56]. As the authors of Band et al. indicate, one 
way of thinking about the approach is that it uses the study-wise estimated log-odds ratio (beta) and its standard error as summary statistics of the data. For each model of association, we assume a prior on the log odds ratio which is normally distributed around zero with a standard deviation of 0.2. By changing the prior on the covariance (or correlation) in effect sizes between studies we can formally compare models where: 1) the effects are independent across studies, and 2) the effects are correlated equally between studies. The final report of results is based on the correlated effects model. To address potential differences in effect sizes for the reported associated variants, we assessed heterogeneity of effects and did not find evidence $\left(\log _{10} \mathrm{BF}>2\right)$. For each model we can obtain a Bayes factor $(\mathrm{BF})$ for association by comparing it with the null model where all the prior weight is on an effect size of zero. We report p-value approximation using the Bayes factor as a statistic for model 2 where the effects are correlated between studies.

Association statistics were combined based on association analysis across three study groups: 1) AJ (1,855 CD and 3,044 non-IBD samples); 2) NFE (2,296 CD and 2,770 non-IBD); and 3) Finnish (FINN) (210 CD and 9,930 non-IBD samples) for a total of 4,361 CD samples and 15,744 non-IBD samples.

Conditional haplotype based testing and variable selection for NOD2 alleles. In the conditional haplotype-based testing (-chap) analysis we used PLINK v1.08p[54] and set a minimum haplotype frequency of .001 ( $-\mathrm{mhf}$ ). We used PLINKSEQ (https://atgu.mgh. harvard.edu/plinkseq/), an open-source $\mathrm{C} / \mathrm{C}++$ library for working with human genetic variation data, and the Python bindings implemented in pyPLINKSEQ to perform Bayesian Model Averaging (BMA). We applied BMA[32] using the R package 'BMA' (https://cran.r-project. org/web/packages/BMA/BMA.pdf).

Polygenic risk scores. The polygenic risk scores were calculated for the international inflammatory bowel diseases consortium European samples. Details of these samples including the QC procedures were described in previous publications[37]. We used reported effect size estimates from $124 \mathrm{CD}$ alleles including those reported in a previously published study [36] and four variants in IL23R from a recent fine-mapping study [37], and excluding variants in NOD2 and LRRK2. We used $454 \mathrm{AJ}$ controls; 1,938 AJ CD; 35,007 non-Jewish controls and 20,652 non-Jewish CD samples. Polygenic risk scores were calculated using array genotype data as the sum of the log odds ratio of the variants associated with CD. Scores for missing genotypes were replaced by the imputed expected value using PLINK[54]. Variants in NOD2 and $L R R K 2$ were excluded from the analysis to assess whether polygenic signal was independent of those genes.

Let $P R S_{i}$ be the polygenic risk score of individual $i$, assuming additive effects on the logodds scale, i.e.

$$
P R S_{i}=\sum_{m=1}^{M} \widehat{\beta_{m}} G_{i, m}
$$

where $\widehat{\beta_{m}}$ denotes the estimated log odds ratio for variant $m$ and $G_{i, m}$ denotes the genotype dosage of individual $i$ for variant $m$. More specifically, $\widehat{\beta_{m}}$ is the effect size estimate of variant $m$ on a logit scale in conferring risk of $\mathrm{CD}$ in an individual.

In the setting where effects are non-additive, i.e. a genotype-specific effect model,

$$
\operatorname{PRS}_{i}^{*}=\sum_{m=1}^{M}\left[\widehat{\beta_{m}^{\text {Het }}} 1_{[\text {Het }]}+\widehat{\beta_{m}^{\text {Hom }}} 1_{[\text {Hom }]}\right]
$$

For now, we consider the additive scenario, and later we return to the setting where nonadditive effects exist, which is relevant for quantifying the differences in contribution of NOD2 alleles to genetic risk in two populations. 
The estimated expected PRS value for an individual in population $j$ is

$$
\mathbb{E}[\widehat{P R S}]_{j}=\sum_{i=1}^{N_{j}} \frac{P R S_{i}}{N_{j}},
$$

where $N_{j}$ is the number of individuals sampled in population $j$. Substituting equation for $P R S_{i}$ and rearranging terms simplifies the equation as a function of variant frequency:

$$
\begin{gathered}
\mathbb{E}[\widehat{P R S}]_{j}=\sum_{i=1}^{N_{j}} \sum_{m=1}^{M} \frac{\widehat{\beta_{m}} G_{i, m}}{N_{j}}, \\
\mathbb{E}[\widehat{P R S}]_{j}=\sum_{m=1}^{M}\left(\sum_{i=1}^{N_{j}} \frac{\widehat{\beta_{m}} G_{i, m}}{N_{j}}\right), \\
\mathbb{E}[\widehat{P R S}]_{j}=\sum_{m=1}^{M}\left(\widehat{\beta_{m}} \sum_{i=1}^{N_{j}} \frac{G_{i, m}}{N_{j}}\right),
\end{gathered}
$$

where $\sum_{i=1}^{N_{j}} \frac{G_{i, m}}{N_{j}}=\widehat{f_{m, j}}$ and $\widehat{f_{m, j}}$ denotes the frequency of variant $m$ in population $j$. Thus, the estimated expected PRS value of an individual in population $j$ is $\mathbb{E}[\widehat{P R S}]_{j}=\sum_{m=1}^{M}\left(2 \widehat{\beta_{m}} \widehat{f_{m, j}}\right)$.

Assume that we are interested in the expected difference in contribution of the studied variants to the PRS between two individuals, say from population 1 being AJ and population 2 being NFE. Also, assume that the effect size of variant $m$ is shared across both populations. Then, using the estimated expected PRS value we define estimated expected difference in contribution of the studied variants to the PRS as the difference in estimated expected PRS value in two populations:

$$
\begin{gathered}
\mathbb{E}[\text { Difference } P R S]=\mathbb{E}[\widehat{P R S}]_{A J}-\mathbb{E}[\widehat{P R S}]_{N F E}, \\
\mathbb{E}[\text { Difference } P R S]=\sum_{m=1}^{M} 2 \widehat{\beta_{m}}\left(\widehat{f_{m, A J}}-\widehat{f_{m, N F E}}\right),
\end{gathered}
$$

which can be used to get an estimated difference in contribution of a variant $m$ to the polygenic risk score in two populations,

$$
\mathbb{E}[\text { Difference } P R S]_{m}=2 \widehat{\beta_{m}}\left(\widehat{f_{m, A J}}-\widehat{f_{m, N F E}}\right) .
$$

To rank variants according to their relative differences in contribution to genetic risk we included the NOD2 and LRRK2 alleles, used the list of estimated effect size from the published studies[36,37], and estimates from this study.

If we substitute $P R S^{*}$ for $P R S$,

$$
\begin{gathered}
\mathbb{E}\left[\widehat{P R S^{*}}\right]_{j}=\sum_{m=1}^{M} \frac{\left(\sum_{i=1}^{N_{j}}\left[\widehat{\beta_{m}^{\mathrm{Het}}} 1_{[\mathrm{Het}]}+\widehat{\beta_{m}^{\mathrm{Hom}}} 1_{[\mathrm{Hom}]}\right]\right)}{N_{j}} \\
=\sum_{m=1}^{M}\left[2 \widehat{f_{m}}\left(1-\widehat{f_{m}}\right) \widehat{\beta_{m}^{\mathrm{Het}}}+{\widehat{f_{m}}}^{2} \widehat{\beta_{m}^{\mathrm{Hom}}}\right] .
\end{gathered}
$$


Then, the estimated expected difference in $P R S^{*}$ when non-additive effects exist is

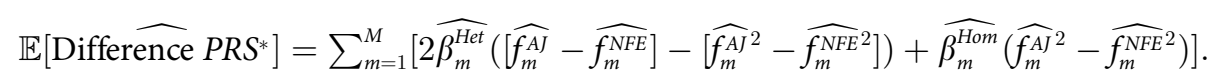

\section{Estimating fold difference in prevalence for a population with shift in expected genetic risk}

Assuming log-additive effects in the logit risk model the disease probability for an individual is given as $p=(1+\exp (-\eta))^{-1}$, where $\eta$ tends towards a normal distribution with parameters $\mu=\log \left(p_{0} /\left(1-p_{0}\right)\right)+\sum_{m=1}^{M} 2 f_{m} \beta_{m}$ and $\sigma^{2}=2 \sum_{m=1}^{M} f_{m}\left(1-f_{m}\right) \beta_{m}^{2}$ [35]. Here $p_{0}$ refers to a baseline disease probability.

We can see that $\mu$ may be expressed in terms of the expected polygenic risk score, i.e. $\mu=\log \left(p_{0} /\left(1-p_{0}\right)\right)+\mathbb{E}[P R S]$. In the setting where $\mathbb{E}[P R S]=0$, then

$$
\mathbb{E}[p]=\left(1+\exp \left(-\log \left(p_{0} /\left(1-p_{0}\right)\right)\right)\right)^{-1}=p_{0} .
$$

To evaluate the impact of a shift in the expected value of polygenic risk score to the expected value of $\mu$ we can express the shift as $\mathbb{E}[$ Difference $\mu]=\mathbb{E}[$ Difference $P R S]$. We can compute new values of $p$ for new values of $\mu$ to obtain a fold-increase in prevalence for a population that has undergone such a shift.

We see that this requires a value to be chosen for $p_{0}$ and that $\log \left(p_{0} /\left(1-p_{0}\right)\right)$ can be represented as a baseline risk score value $\beta_{0}$. To get an estimate of the absolute prevalence of CD in the AJ population, we must choose a baseline $\beta_{0}$, where $p_{0}$ represents the expected prevalence with zero non-baseline alleles in the population [35], to which we add a contribution from multiple non-baseline alleles to calculate: 1) an individual's probability of disease, or 2) the expected prevalence of the disease in the population.

Once we have chosen a value for $\beta_{0}$, we can calculate the ratio of expected prevalence as follows. First, use the means $\left(\mu_{\mathrm{AJ}}\right.$ and $\left.\mu_{\mathrm{NAJ}}\right)$ and variances $\left(\sigma_{A J}^{2}\right.$ and $\left.\sigma_{N A J}^{2}\right)$ of risk scores as calculated above to calculate the probability density function of the disease prevalence. In the case of the AJ population, we have

$$
f(p)=\frac{d n}{d g} \frac{1}{\sigma_{A J}} \phi\left(\frac{\eta-\mu_{A J}}{\sigma_{A J}}\right)=\frac{1}{\sigma_{A J} p(1-p)} \phi\left(\frac{1}{\sigma_{A J}} \log \left(\frac{p}{1-p}\right)-\frac{\mu_{A J}}{\sigma_{A J}}\right)
$$

where $\eta$ is the risk score associated with prevalence $p, g$ is the link function, so $p=g(\eta)=(1+$ $\left.e^{-\eta}\right)^{-1}$, and $\phi$ is the standard normal density function.

Next, we integrate to get $\int_{0}^{1} p \cdot f(p) d p=\mathbb{E}\left[p_{A J}\right]$. Finally, we can calculate $\mathbb{E}\left[p_{N A J}\right]$ in a similar way, and divide the expected prevalence in the AJ population by that in the non-AJ population to get the prevalence ratio, $\mathbb{E}\left[p_{A J}\right] / \mathbb{E}\left[p_{N A J}\right]$.

The value of $\beta_{0}=-20.5$ was chosen in order to obtain a prevalence in the non-AJ population of $\sim 0.5 \%$. At this value of $\beta_{0}$, the ratio of prevalence in the AJ population to that in the non-AJ population was estimated to be $1.5\left(\mathbb{E}\left[p_{A J}\right]=0.82 \%, \mathbb{E}\left[p_{N A J}\right]=0.55 \%\right)$.

For different choices of $\beta_{0}$, however, this ratio may vary, as the relationship between probability of disease and risk score is non-linear. S10 Fig shows how the values of the disease prevalence and their ratio vary as $\beta_{0}$ is changed. We see that the ratio values range from 1.46 to 1.52 for different values of $\beta_{0}$ with a range of baseline prevalence of .001 to .01-the range of prevalence estimates for Crohn's disease[41,43,57].

To further understand the effect that choosing a logit-based model had on the results, a comparison of the standard logit and probit models was done using the values inferred from 
the logit model. No full scale probit modelling was done in this analysis, so the values found with the probit model represent only a close approximation of the expected results.

In the logit model for population analysis, we may assume that individual risk scores are chosen from a normal distribution $\mathcal{N}\left(\mu_{\text {logit }}, \sigma_{\text {logit }}^{2}\right)$ where $\mu_{\text {logit }}$ and $\sigma_{\text {logit }}$ represent the mean and standard deviation of the risk scores as defined above. From here, we may calculate the probability density function of probit model risk scores $\mu_{\text {probit }}$ based on that of logit model risk scores $\mu_{\text {logit }}$ as

$$
f\left(\eta_{\text {probit }} \mid \mu_{\text {logit }}, \sigma_{\text {logit }}\right)=f\left(\eta_{\text {logit }} \mid \mu_{\text {logit }}, \sigma_{\text {logit }}\right) d \eta_{\text {logit }} / d \eta_{\text {probit }}
$$

and use this to calculate $\mu_{\text {probit }}$ and $\sigma_{\text {probit }}$, the estimated mean and standard deviation of the risk scores in the probit model. Using these values, we obtain a probability distribution for the frequency of disease in the populations using the probit model.

While the logit model yielded a prevalence ratio of 1.506 , the probit estimation yielded a prevalence ratio of 1.5136 , with similar expected prevalence values $\left(\mathbb{E}\left[p_{A J}\right]=0.823 \%, \mathbb{E}\left[p_{N A]}\right]=\right.$ $0.544 \%)$. These values demonstrate that individual logit and probit analyses would likely give similar results for values of interest. The complete probability densities under the logit and probit models can be seen in S8 Fig.

Further, it is interesting to compare the relationship between values of risk scores in the two models. For values of risk scores between -1 and 1 in the logit model, the relationship to those in the probit model is highly linear, with a formula of $\eta_{\text {probit }}=0.6223 \cdot \eta_{\text {logit }}$, with $\mathrm{r}^{2}=$ 1.0000. This formula may be used to impute single values in one model or the other assuming that the estimated total risk score is otherwise close to zero, and the imputed value is low. It is worth noting, however, that this does not work for all values of $\eta_{\text {logit }}$ as the relationship between risk score in the logit and probit models deviates from this simple linear model when the risk score values are large.

Difference in prevalence between $\mathrm{AJ}$ and NFE attributed to implicated variants. The difference in prevalence due to multiple alleles can be computed as

$$
\text { Prevalence difference }=\frac{\left(\left(p_{2}-p_{1}\right)-\left(i_{2}-i_{1}\right)\right)}{\left(p_{2}-p_{1}\right)},
$$

where $p_{j}$ denotes the disease prevalence in population $j$ and $i_{j}$ denotes the disease prevalence without the risk factors in population $j$, which according to Moonesinghe et al.[58] is

$$
i_{j}=\frac{p_{j}}{\prod_{m=1}^{M}\left(1+f_{m, j}\left(G R R_{m}-1\right)\right)^{2}}
$$

where $G R R_{m}$ denotes the genotype relative risk for variant $m$.

We model the $\mathrm{CD}$ prevalence accounted for by $\mathrm{CD}$ associated enriched protein-altering alleles separately in both AJ and non-AJ European and determine the amount that CD prevalence would be reduced if this variant were absent from each population.

To estimate the difference in prevalence between two populations attributed to genetic risk factors when non-additive effects exist,

$$
i_{j}=\frac{p_{j}}{\prod_{m=1}^{M}\left(1+2 f_{m, j}\left(G R R_{m}^{\text {Het }}-1\right)+f_{m, j}^{2}\left(G R R_{m}^{\text {Hom }}-1\right)\right)} .
$$




\section{Enirchment testing sensitivity}

When modeling enrichment, we chose a standard significance cutoff of $\mathrm{p}<0.05 / \mathrm{N}$ for classifying variants as enriched. We noted that the number of variants classified as enriched does not change significantly when the p-value threshold changes. See S11 Fig for more information.

\section{Supporting information}

S1 Fig. Analysis workflow diagram.

(PNG)

S2 Fig. Admixture plots.

(PNG)

S3 Fig. Cross-validation errors for number of clusters in ADMIXTURE.

(PNG)

S4 Fig. MAF thresholds chosen for enrichment testing. (PNG)

S5 Fig. Principal components plot for 5,685 AJ individuals.

(PNG)

S6 Fig. Variable selection using Bayesian model averaging (BMA).

(PNG)

S7 Fig. AJ individuals have higher CD polygenic risk score than NJ controls. (PNG)

S8 Fig. Dependence of population prevalence on $\boldsymbol{\beta}_{0}$. (PNG)

S9 Fig. Probit and logit model analysis.

(PNG)

S10 Fig. The relationship between expected differences in genetic risk score and expected fold differences in disease prevalence.

(PNG)

S11 Fig. Sensitivity test for p-value significance cutoff.

S1 Data File. ClinVar pathogenic alleles enriched in AJ.

(XLSX)

S2 Data File. AJ enrichment data for all analyzed alleles.

S1 Table. Origins of moderate ancestry fraction Ashkenazi samples.

(PNG)

S2 Table. Origins of high ancestry fraction Ashkenazi samples.

(PNG)

S3 Table. Conditional haplotype-based testing in NOD2.

(PNG) 
S4 Table. Assessing association of a one-hit and two-hit model of NOD2 in the AJ exome sequencing data.

(PNG)

S5 Table. Assessing association of a one-hit and two-hit model of NOD2 in the non-AJ immunoChip data.

(JPG)

\section{Acknowledgments}

Manuel A. Rivas is a Faculty Fellow at the Stanford Center for Population Health Sciences. We thank the Broad IT team for assistance with the IBD exomes browser.

\section{NIDDK IBD Genetics Consortium}

Abraham C, Achkar JP, Bitton A, Boucher G, Croitoru K, Fleshner P, Kugathasan S, Limbergen JV, Milgrom R, Proctor D, Regueiro M, Schumm PL, Sharma Y, Stempak JM, Targan SR, Wang $\mathrm{MH}$.

\section{International IBD Genetics Consortium}

Clara Abraham, Jean-Paul Achkar, Tariq Ahmad, Leila Amininejad, Ashwin N Ananthakrishnan, Vibeke Andersen, Carl A Anderson, Jane M Andrews, Vito Annese, Guy Aumais, Leonard Baidoo, Robert N Baldassano, Peter A Bampton, Murray Barclay, Jeffrey C Barrett, Theodore M Bayless, Johannes Bethge, Alain Bitton, Gabrielle Boucher, Stephan Brand, Berenice Brandt, Steven R Brant, Carsten Büning, Angela Chew, Judy H Cho, Isabelle Cleynen, Ariella Cohain, Anthony Croft, Mark J Daly, Mauro D’Amato, Silvio Danese, Dirk De Jong, Martine De Vos, Goda Denapiene, Lee A Denson, Kathy L Devaney, Olivier Dewit, Renata D’Inca, Marla Dubinsky, Richard H Duerr, Cathryn Edwards, David Ellinghaus, Jonah Essers, Lynnette R Ferguson, Eleonora A Festen, Philip Fleshner, Tim Florin, Denis Franchimont, Andre Franke, Karin Fransen, Richard Gearry, Michel Georges, Christian Gieger, Jürgen Glas, Philippe Goyette, Todd Green, Anne M Griffiths, Stephen L Guthery, Hakon Hakonarson, Jonas Halfvarson, Katherine Hanigan, Talin Haritunians, Ailsa Hart, Chris Hawkey, Nicholas K Hayward, Matija Hedl, Paul Henderson, Xinli Hu, Hailiang Huang, Jean-Pierre Hugot, Ken Y Hui, Marcin Imielinski, Andrew Ippoliti, Laimas Jonaitis, Luke Jostins, Tom H Karlsen, Nicholas A Kennedy, Mohammed Azam Khan, Gediminas Kiudelis, Krupa Krishnaprasad, Subra Kugathasan, Limas Kupcinskas, Anna Latiano, Debby Laukens, Ian C Lawrance, James C Lee, Charlie W Lees, Marcis Leja, Johan Van Limbergen, Paolo Lionetti, Jimmy Z Liu, Edouard Louis, Gillian Mahy, John Mansfield, Dunecan Massey, Christopher G Mathew, Dermot PB McGovern, Raquel Milgrom, Mitja Mitrovic, Grant W Montgomery, Craig Mowat, William Newman, Aylwin Ng, Siew C Ng, Sok Meng Evelyn Ng, Susanna Nikolaus, Kaida Ning, Markus Nöthen, Ioannis Oikonomou, Orazio Palmieri, Miles Parkes, Anne Phillips, Cyriel Y Ponsioen, Urõs Potocnik, Natalie J Prescott, Deborah D Proctor, Graham RadfordSmith, Jean-Francois Rahier, Soumya Raychaudhuri, Miguel Regueiro, Florian Rieder, John D Rioux, Stephan Ripke, Rebecca Roberts, Richard K Russell, Jeremy D Sanderson, Miquel Sans, Jack Satsangi, Eric E Schadt, Stefan Schreiber, Dominik Schulte, L Philip Schumm, Regan Scott, Mark Seielstad, Yashoda Sharma, Mark S Silverberg, Lisa A Simms, Jurgita Skieceviciene, Sarah L Spain, A. Hillary Steinhart, Joanne M Stempak, Laura Stronati, Jurgita Sventoraityte, Stephan R Targan, Kirstin M Taylor, Anje ter Velde, Emilie Theatre, Leif Torkvist, Mark Tremelling, Andrea van der Meulen, Suzanne van Sommeren, Eric Vasiliauskas, Severine Vermeire, Hein W Verspaget, Thomas Walters, Kai Wang, Ming-Hsi Wang, Rinse K 
Weersma, Zhi Wei, David Whiteman, Cisca Wijmenga, David C Wilson, Juliane Winkelmann, Ramnik J Xavier, Bin Zhang, Clarence K Zhang, Hu Zhang, Wei Zhang, Hongyu Zhao, Zhen Z Zhao.

\section{T2D-GENES Consortium}

Christian Fuchsberger, Jason Flannick, Tanya M Teslovich, Anubha Mahajan, Vineeta Agarwala, Kyle J Gaulton, Clement Ma, Pierre Fontanillas, Loukas Moutsianas, Davis J McCarthy, Manuel A Rivas, John R B Perry, Xueling Sim, Thomas W Blackwell, Neil R Robertson, William Rayner, Pablo Cingolani, Adam E Locke, Juan Fernandez Tajes, Heather M Highland, Josee Dupuis, Peter S Chines, Cecilia M Lindgren, Christopher Hartl, Anne U Jackson, Han Chen, Jeroen R Huyghe, Martijn van de Bunt, Richard D Pearson, Ashish Kumar, Martina Müller-Nurasyid, Niels Grarup, Heather M Stringham, Eric R Gamazon, Jaehoon Lee, Yuhui Chen, Robert A Scott, Jennifer E Below, Peng Chen, Jinyan Huang, Min Jin Go, Michael L Stitzel, Dorota Pasko, Stephen C J Parker, Tibor V Varga, Todd Green, Nicola L Beer, Aaron G Day Williams, Teresa Ferreira, Tasha Fingerlin, Momoko Horikoshi, Cheng Hu, Iksoo Huh, Mohammad Kamran Ikram, Bong Jo Kim, Yongkang Kim, Young Jin Kim, Min Seok Kwon, Juyoung Lee, Selyeong Lee, Keng Han Lin, Taylor J Maxwell, Yoshihiko Nagai, Xu Wang, Ryan P Welch, Joon Yoon, Weihua Zhang, Nir Barzilai, Benjamin F Voight, Bok Ghee Han, Christopher P Jenkinson, Teemu Kuulasmaa, Johanna Kuusisto, Alisa Manning, Maggie C Y Ng, Nicholette D Palmer, Beverley Balkau, Alena Stančáková, Hanna E Abboud, Heiner Boeing, Vilmantas Giedraitis, Dorairaj Prabhakaran, Omri Gottesman, James Scott, Jason Carey, Phoenix Kwan, George Grant, Joshua D Smith, Benjamin M Neale, Shaun Purcell, Adam S Butterworth, Joanna M Howson, Heung Man Lee, Yingchang Lu, Soo Heon Kwak, Wei Zhao, John Danesh, Vincent K L Lam, Kyong Soo Park, Danish Saleheen, Wing Yee So, Claudia H T Tam, Uzma Afzal, David Aguilar, Rector Arya, Tin Aung, Edmund Chan, Carmen Navarro, Ching Yu Cheng, Domenico Palli, Adolfo Correa, Joanne E Curran, Denis Rybin, Vidya S Farook, Sharon P Fowler, Barry I Freedman, Michael Griswold, Daniel Esten Hale, Pamela J Hicks, Chiea-Chuen Khor, Satish Kumar, Benjamin Lehne, Dorothée Thuillier, Wei Yen Lim, Jianjun Liu, Yvonne T van der Schouw, Marie Loh, Solomon K Musani, Sobha Puppala, William R Scott, Loïc Yengo, Sian Tsung Tan, Herman A Taylor Jr, Farook Thameem, Gregory Wilson Sr, Tien Yin Wong, Pål Rasmus Njølstad, Jonathan C Levy, Massimo Mangino, Lori L Bonnycastle, Thomas Schwarzmayr, João Fadista, Gabriela L Surdulescu, Christian Herder, Christopher J Groves, Thomas Wieland, Jette Bork Jensen, Ivan Brandslund, Cramer Christensen, Heikki A Koistinen, Alex S F Doney, Leena Kinnunen, Tõnu Esko, Andrew J Farmer, Liisa Hakaste, Dylan Hodgkiss, Jasmina Kravic, Valeriya Lyssenko, Mette Hollensted, Marit E Jørgensen, Torben Jørgensen, Claes Ladenvall, Johanne Marie Justesen, Annemari Käräjämäki, Jennifer Kriebel, Wolfgang Rathmann, Lars Lannfelt, Torsten Lauritzen, Narisu Narisu, Allan Linneberg, Olle Melander, Lili Milani, Matt Neville, Marju Orho Melander, Lu Qi, Qibin Qi, Michael Roden, Olov Rolandsson, Amy Swift, Anders H Rosengren, Kathleen Stirrups, Andrew R Wood, Evelin Mihailov, Christine Blancher, Mauricio O Carneiro, Jared Maguire, Ryan Poplin, Khalid Shakir, Timothy Fennell, Mark DePristo, Martin Hrabé de Angelis, Panos Deloukas, Anette P Gjesing, Goo Jun, Peter Nilsson, Jacquelyn Murphy, Robert Onofrio, Barbara Thorand, Torben Hansen, Christa Meisinger, Frank B Hu, Bo Isomaa, Fredrik Karpe, Liming Liang, Annette Peters, Cornelia Huth, Stephen P O’Rahilly, Colin N A Palmer, Oluf Pedersen, Rainer Rauramaa, Jaakko Tuomilehto, Veikko Salomaa, Richard M Watanabe, Ann Christine Syvänen, Richard N Bergman, Dwaipayan Bharadwaj, Erwin P Bottinger, Yoon Shin Cho, Giriraj R Chandak, Juliana C N Chan, Kee Seng Chia, Mark J Daly, Shah B Ebrahim, Claudia Langenberg, Paul Elliott, Kathleen A Jablonski, Donna M Lehman, 
Weiping Jia, Ronald C W Ma, Toni I Pollin, Manjinder Sandhu, Nikhil Tandon, Philippe Froguel, Inês Barroso, Yik Ying Teo, Eleftheria Zeggini, Ruth J F Loos, Kerrin S Small, Janina S Ried, Ralph A DeFronzo, Harald Grallert, Benjamin Glaser, Andres Metspalu, Nicholas J Wareham, Mark Walker, Eric Banks, Christian Gieger, Erik Ingelsson, Hae Kyung Im, Thomas Illig, Paul W Franks, Gemma Buck, Joseph Trakalo, David Buck, Inga Prokopenko, Reedik Mägi, Lars Lind, Yossi Farjoun, Katharine R Owen, Anna L Gloyn, Konstantin Strauch, Tiinamaija Tuomi, Jaspal Singh Kooner, Jong Young Lee, Taesung Park, Peter Donnelly, Andrew D Morris, Andrew T Hattersley, Donald W Bowden, Francis S Collins, Gil Atzmon, John C Chambers, Timothy D Spector, Markku Laakso, Tim M Strom, Graeme I Bell, John Blangero, Ravindranath Duggirala, E Shyong Tai, Gilean McVean, Craig L Hanis, James G Wilson, Mark Seielstad, Timothy M Frayling, James B Meigs, Nancy J Cox, Rob Sladek, Eric S Lander, Stacey Gabriel, Noël P Burtt, Karen L Mohlke, Thomas Meitinger, Leif Groop, Goncalo Abecasis, Jose C Florez, Laura J Scott, Andrew P Morris, Hyun Min Kang, Michael Boehnke, David Altshuler, Mark I McCarthy.

\section{Author Contributions}

Conceptualization: Manuel A. Rivas, Judy H. Cho, Dermot P. B. McGovern, Mark J. Daly.

Data curation: Manuel A. Rivas, Hailiang Huang, Christine Stevens, Matti Pirinen, Benjamin M. Neale, Mitja Kurki, Andrea Ganna, Monkol Lek, Konrad J. Karczewski, Aarno Palotie, John D. Rioux, Rinse K. Weersma, Andre Franke, Jeffrey C. Barrett, Daniel G. MacArthur.

Formal analysis: Manuel A. Rivas, Brandon E. Avila, Jukka Koskela, Hailiang Huang, Matti Pirinen, Monkol Lek, Mark J. Daly.

Funding acquisition: Mark J. Daly.

Investigation: Manuel A. Rivas, Judy H. Cho, Dermot P. B. McGovern, Mark J. Daly.

Methodology: Manuel A. Rivas, Brandon E. Avila, Hailiang Huang, Matti Pirinen, Mark J. Daly.

Project administration: Christine Stevens, Mark J. Daly.

Resources: Christine Stevens, Talin Haritunians, Benjamin M. Neale, Daniel Graham, Benjamin Glaser, Inga Peter, Gil Atzmon, Nir Barzilai, Adam P. Levine, Elena Schiff, Nikolas Pontikos, Ben Weisburd, Konrad J. Karczewski, Eric V. Minikel, Britt-Sabina Petersen, Laurent Beaugerie, Philippe Seksik, Jacques Cosnes, Stefan Schreiber, Bernd Bokemeyer, Johannes Bethge, Graham Heap, Tariq Ahmad, Vincent Plagnol, Anthony W. Segal, Stephan Targan, Dan Turner, Paivi Saavalainen, Martti Farkkila, Kimmo Kontula, Aarno Palotie, Steven R. Brant, Richard H. Duerr, Mark S. Silverberg, John D. Rioux, Rinse K. Weersma, Andre Franke, Luke Jostins, Carl A. Anderson, Jeffrey C. Barrett, Daniel G. MacArthur, Chaim Jalas, Harry Sokol, Ramnik J. Xavier, Ann Pulver, Judy H. Cho, Dermot P. B. McGovern, Mark J. Daly.

Software: Manuel A. Rivas, Brandon E. Avila, Jonathan Bloom.

Supervision: Mark J. Daly.

Validation: Brandon E. Avila, Jukka Koskela.

Visualization: Manuel A. Rivas, Brandon E. Avila, Jukka Koskela, Hailiang Huang, Mark J. Daly.

Writing - original draft: Manuel A. Rivas. 
Writing - review \& editing: Brandon E. Avila, Jukka Koskela, Judy H. Cho, Dermot P. B. McGovern, Mark J. Daly.

\section{References}

1. Ostrer H. \& Skorecki K. The population genetics of the Jewish people. Hum. Genet. 132, 119-127 (2012). https://doi.org/10.1007/s00439-012-1235-6 PMID: 23052947

2. Moltke I., Grarup N., Jørgensen M. E., Bjerregaard P., Treebak J.T., Fumagalli M., et al. A common Greenlandic TBC1D4 variant confers muscle insulin resistance and type 2 diabetes. Nature 512, 190193 (2014). https://doi.org/10.1038/nature13425 PMID: 25043022

3. Lim E. T., Würtz P., Havulinna A. S., Palta P., Tukiainen T., Rehnström K., et al. Distribution and medical impact of loss-of-function variants in the Finnish founder population. PLoS Genet. 10, e1004494 (2014). https://doi.org/10.1371/journal.pgen.1004494 PMID: 25078778

4. Zuk O., Schaffner S. F., Samocha K., Do R., Hechter E., Kathiresan S., et al. Searching for missing heritability: designing rare variant association studies. Proc. Natl. Acad. Sci. U. S. A. 111, E455-64 (2014). https://doi.org/10.1073/pnas.1322563111 PMID: 24443550

5. Bahcall O. \& Orli B. Rare variant association studies. Nat. Genet. 46, 219-219 (2014).

6. Kenny E. E., Pe'er I., Karban A., Ozelius L., Mitchell A. A., Ng S.M., et al. A genome-wide scan of Ashkenazi Jewish Crohn's disease suggests novel susceptibility loci. PLoS Genet. 8, e1002559 (2012). https://doi.org/10.1371/journal.pgen.1002559 PMID: 22412388

7. Karban A., Eliakim R. \& Brant S. R. Genetics of inflammatory bowel disease. Isr. Med. Assoc. J. 4, 798-802 (2002). PMID: 12389344

8. Baskovich B., Hiraki S., Upadhyay K., Meyer P., Carmi S., Barzilai N., et al. Expanded genetic screening panel for the Ashkenazi Jewish population. Genet. Med. 18, 522-528 (2016). https://doi.org/10. 1038/gim.2015.123 PMID: 26334176

9. Lek M., Karczewski K. J., Minikel E. V., Samocha K. E., Banks E., Fennell T., et al. Analysis of proteincoding genetic variation in 60,706 humans. Nature 536, 285-291 (2016). https://doi.org/10.1038/ nature19057 PMID: 27535533

10. Fuchsberger C., Flannick J., Teslovich T. M., Mahajan A., Agarwala V., Gaulton K. J., et al. The genetic architecture of type 2 diabetes. Nature 536, 41-47 (2016). https://doi.org/10.1038/nature18642 PMID: 27398621

11. Karczewski K. J., Weisburd B., Thomas B., Solomonson M., Ruderfer D. M., Kavanagh D., et al. The ExAC Browser: Displaying reference data information from over 60,000 exomes. (2016). https://doi.org/ 10.1101/070581

12. Alexander D. H., Novembre J. \& Lange K. Fast model-based estimation of ancestry in unrelated individuals. Genome Res. 19, 1655-1664 (2009). https://doi.org/10.1101/gr.094052.109 PMID: 19648217

13. Mathieson I. \& McVean G. Demography and the Age of Rare Variants. PLoS Genet. 10, e1004528 (2014). https://doi.org/10.1371/journal.pgen.1004528 PMID: 25101869

14. Landrum M. J., Lee J. M., Benson M., Brown G., Chao C., Chitipiralla S., et al. ClinVar: public archive of interpretations of clinically relevant variants. Nucleic Acids Res. 44, D862-D868 (2015). https://doi.org/ 10.1093/nar/gkv1222 PMID: 26582918

15. Gross S. J., Pletcher B. A., Monaghan K. G. \& Professional Practice and Guidelines Committee. Carrier screening in individuals of Ashkenazi Jewish descent. Genet. Med. 10, 54-56 (2008). https://doi.org/ 10.1097/GIM.0b013e31815f247c PMID: 18197057

16. Chang W., Winder T. L., LeDuc C. A., Simpson L. L., Millar W. S., Dungan J. et al. Founder Fukutin mutation causes Walker-Warburg syndrome in four Ashkenazi Jewish families. Prenat. Diagn. 29, 560-569 (2009). https://doi.org/10.1002/pd.2238 PMID: 19266496

17. Fedick A. M., Jalas C., Treff N. R., Knowles M. R. \& Zariwala M. A. Carrier frequencies of eleven mutations in eight genes associated with primary ciliary dyskinesia in the Ashkenazi Jewish population. Mol Genet Genomic Med 3, 137-142 (2015). https://doi.org/10.1002/mgg3.124 PMID: 25802884

18. Edvardson S., Shaag A., Zenvirt S., Erlich Y., Hannon G. J., Shanske A. L., et al. Joubert syndrome 2 (JBTS2) in Ashkenazi Jews is associated with a TMEM216 mutation. Am. J. Hum. Genet. 86, 93-97 (2010). https://doi.org/10.1016/j.ajhg.2009.12.007 PMID: 20036350

19. Edvardson S., Kose S., Jalas C., Fattal-Valevski A., Watanabe A., Ogawa Y., et al. Leukoencephalopathy and early death associated with an Ashkenazi-Jewish founder mutation in the Hikeshi gene. J. Med. Genet. 53, 132-137 (2016). https://doi.org/10.1136/jmedgenet-2015-103232 PMID: 26545878 
20. Fedick A., Jalas C. \& Treff N. R. A deleterious mutation in the PEX2 gene causes Zellweger syndrome in individuals of Ashkenazi Jewish descent. Clin. Genet. 85, 343-346 (2014). https://doi.org/10.1111/ cge.12170 PMID: 23590336

21. Edvardson S., Gerhard F., Jalas C., Lachmann J., Golan D., Saada A., et al. Hypomyelination and developmental delay associated with VPS11 mutation in Ashkenazi-Jewish patients. J. Med. Genet. 52, 749-753 (2015). https://doi.org/10.1136/jmedgenet-2015-103239 PMID: 26307567

22. Fedick A., Jalas C., Abeliovich D., Krakinovsky Y., Ekstein J., Ekstein A., et al. Carrier frequency of two BBS2 mutations in the Ashkenazi population. Clin. Genet. 85, 578-582 (2014). https://doi.org/10.1111/ cge.12231 PMID: 23829372

23. Gershoni-Baruch R., Broza Y. \& Brik R. Prevalence and significance of mutations in the familial Mediterranean fever gene in Henoch-Schönlein purpura. J. Pediatr. 143, 658-661 (2003). https://doi.org/10. 1067/S0022-3476(03)00502-X PMID: 14615741

24. Rivas M. A., Graham D., Sulem P., Stevens C., Desch A. N., Goyette P., et al. A protein-truncating R179X variant in RNF186 confers protection against ulcerative colitis. Nat. Commun. 7, 12342 (2016). https://doi.org/10.1038/ncomms12342 PMID: 27503255

25. Firth D. \& D., F. 'Bias reduction of maximum likelihood estimates'. Biometrika 82, 667-667 (1995).

26. Ma C., Blackwell T., Boehnke M., Scott L. J. \& GoT2D investigators. Recommended joint and metaanalysis strategies for case-control association testing of single low-count variants. Genet. Epidemiol. 37, 539-550 (2013). https://doi.org/10.1002/gepi.21742 PMID: 23788246

27. Kang H. M. EPACTS: efficient and parallelizable association container toolbox. (2012).

28. Band G., Le Q. S., Jostins L., Pirenin M., Kivinen K., Jallow M., Sisay-Joof F., et al. Imputation-based meta-analysis of severe malaria in three African populations. PLoS Genet. 9, e1003509 (2013). https:// doi.org/10.1371/journal.pgen.1003509 PMID: 23717212

29. Rivas M. A., Beaudoin M., Gardet A., Stevens C., Sharma Y., Zhang C., et al. Deep resequencing of GWAS loci identifies independent rare variants associated with inflammatory bowel disease. Nat. Genet. 43, 1066-1073 (2011). https://doi.org/10.1038/ng.952 PMID: 21983784

30. Liu J. Z., van Sommeren S., Huang H., Ng S. C., Alberts R., Takahashi A., et al. Association analyses identify 38 susceptibility loci for inflammatory bowel disease and highlight shared genetic risk across populations. Nat. Genet. 47, 979-986 (2015). https://doi.org/10.1038/ng.3359 PMID: 26192919

31. Ozelius L. J., Senthil G., Saunders-Pullman R., Ohmann E., Deligtisch A., Tagliati M., et al. LRRK2 G2019S as a cause of Parkinson's disease in Ashkenazi Jews. N. Engl. J. Med. 354, 424-425 (2006). https://doi.org/10.1056/NEJMc055509 PMID: 16436782

32. Raftery A. E. Bayesian Model Selection in Social Research. Sociol. Methodol. 25, 111 (1995).

33. Zhang Q., Pan Y., Yan R., Zeng B., Wang H., Zhang X., et al. Commensal bacteria direct selective cargo sorting to promote symbiosis. Nat. Immunol. 16, 918-926 (2015). https://doi.org/10.1038/ni.3233 PMID: 26237551

34. Ogura Y., Bonen D.K., Inohara N., Nicolae D. L., Chen F. F., Ramos R., et al. A frameshift mutation in NOD2 associated with susceptibility to Crohn's disease. Nature 411, 603-606 (2001). https://doi.org/ 10.1038/35079114 PMID: 11385577

35. Jostins L. Using next-generation genomic datasets in disease association. ( University of Cambridge, 2013).

36. Jostins L., Ripke S., Weersma R. K., Duerr R. H., McGovern D. P., Hui K. Y., et al. Host-microbe interactions have shaped the genetic architecture of inflammatory bowel disease. Nature 491, 119-124 (2012). https://doi.org/10.1038/nature11582 PMID: 23128233

37. Huang H., Fang M., Jostins L., Umićević Mirkov M., Boucher G., Anderson C. A., et al. Association mapping of inflammatory bowel disease loci to single variant resolution. (2015). https://doi.org/10.1101/ 028688

38. Ripke S., Neale B. M., Corvin A., Walters J. T., Farh K. H., Holmans P. A., et al. Biological insights from 108 schizophrenia associated genetic loci. Nature 511, 421-427 (2014) https://doi.org/10.1038/ nature13595 PMID: 25056061

39. Richard M. L., Lamas B., Liguori G., Hoffmann T. W. \& Sokol H. Gut fungal microbiota: the Yin and Yang of inflammatory bowel disease. Inflamm. Bowel Dis. 21, 656-665 (2015). https://doi.org/10.1097/ MIB.0000000000000261 PMID: 25545379

40. Turunen P., Kolho K. L., Auvinen A., Ittanen S., Huhtala H \& Ashorn M. Incidence of inflammatory bowel disease in Finnish children, 1987-2003. Inflamm. Bowel Dis. 12, 677-683 (2006). PMID: 16917221

41. Bernstein C. N., Blanchard J. F., Rawsthorne P. \& Wajda A. Epidemiology of Crohn's Disease and Ulcerative Colitis in a Central Canadian Province: A Population-based Study. Am. J. Epidemiol. 149, 916-924 (1999). PMID: 10342800 
42. Mayberry J. F., Rhodes J. \& Newcombe R. G. Familial prevalence of inflammatory bowel disease in relatives of patients with Crohn's disease. BMJ 280, 84-84 (1980).

43. Sandier R. S. The epidemiology of inflammatory bowel disease. Curr. Opin. Gastroenterol. 6, 531-535 (1990).

44. Nakagome S., Mano S., Kozlowski L., Bujnicki J. M., Shibata H., Fukumaki Y., et al. Crohn's disease risk alleles on the NOD2 locus have been maintained by natural selection on standing variation. Mol. Biol. Evol. 29, 1569-1585 (2012). https://doi.org/10.1093/molbev/mss006 PMID: 22319155

45. Balick D. J., Do R., Cassa C. A., Reich D. \& Sunyaev S. R. Dominance of Deleterious Alleles Controls the Response to a Population Bottleneck. PLoS Genet. 11, e1005436 (2015). https://doi.org/10.1371/ journal.pgen.1005436 PMID: 26317225

46. Schurr E., Erwin S. \& Philippe G. A Common Genetic Fingerprint in Leprosy and Crohn's Disease? N. Engl. J. Med. 361, 2666-2668 (2009). https://doi.org/10.1056/NEJMe0910690 PMID: 20018963

47. Liu H., Irwanto A., Fu X., Yu G., Yu Y., Sun Y., et al. Discovery of six new susceptibility loci and analysis of pleiotropic effects in leprosy. Nat. Genet. 47, 267-271 (2015). https://doi.org/10.1038/ng.3212 PMID: 25642632

48. Zhang F.-R., Huang W., Chen S.-M., Sun L.-D., Liu H., Li Y. et al. Genomewide association study of leprosy. N. Engl. J. Med. 361, 2609-2618 (2009). https://doi.org/10.1056/NEJMoa0903753 PMID: 20018961

49. Turchin M. C., Chiang C. W. K., Palmer C., D., Sankararaman S., Reich D., GIANT Consortium, et al. Evidence of widespread selection on standing variation in Europe at height-associated SNPs. Nat. Genet. 44, 1015-1019 (2012). https://doi.org/10.1038/ng.2368 PMID: 22902787

50. Schizophrenia Working Group of the Psychiatric Genomics Consortium. Biological insights from 108 schizophrenia-associated genetic loci. Nature 511, 421-427 (2014). https://doi.org/10.1038/ nature13595 PMID: 25056061

51. Li H. \& Durbin R. Fast and accurate short read alignment with Burrows-Wheeler transform. Bioinformatics 25, 1754-1760 (2009). https://doi.org/10.1093/bioinformatics/btp324 PMID: 19451168

52. Heliö T., Halme L., Lappalainen M., Fodstad H., Paavola-Sakki P., Turunen U., et al. CARD15/NOD2 gene variants are associated with familially occurring and complicated forms of Crohn's disease. Gut 52, 558-562 (2003). PMID: 12631669

53. Lappalainen M., Paavola-Sakki P., Halme L., Turunen U., Färkkilä M., Repo H., et al. Novel CARD15/ NOD2 mutations in Finnish patients with Crohn's disease and their relation to phenotypic variation in vitro and in vivo. Inflamm. Bowel Dis. 14, 176-185 (2008). https://doi.org/10.1002/ibd.20287 PMID: 17941079

54. Purcell S., Neale B., Todd-Brown K., Thomas L., Ferreira M. A., Bender D., et al. PLINK: a tool set for whole-genome assocMiation and population-based linkage analyses. Am. J. Hum. Genet. 81, 559-575 (2007). https://doi.org/10.1086/519795 PMID: 17701901

55. Tan A., Abecasis G. R. \& Kang H. M. Unified representation of genetic variants. Bioinformatics 31 , 2202-2204 (2015). https://doi.org/10.1093/bioinformatics/btv112 PMID: 25701572

56. Wakefield J. \& Jon W. A Bayesian Measure of the Probability of False Discovery in Genetic Epidemiology Studies. Am. J. Hum. Genet. 81, 208-227 (2007). https://doi.org/10.1086/519024 PMID: 17668372

57. Baumgart D. C. \& Sandborn W. J. Crohn's disease. Lancet 380, 1590-1605 (2012). https://doi.org/10. 1016/S0140-6736(12)60026-9 PMID: 22914295

58. Moonesinghe R., loannidis J. P. A., Flanders W. D., Yang Q., Truman B. \& Khoury M. Estimating the contribution of genetic variants to difference in incidence of disease between population groups. Eur. J. Hum. Genet. 20, 831-836 (2012). https://doi.org/10.1038/ejhg.2012.15 PMID: 22333905 


\section{University Library}

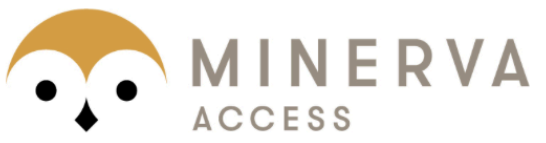

A gateway to Melbourne's research publications

Minerva Access is the Institutional Repository of The University of Melbourne

\section{Author/s:}

Rivas, MA;Avila, BE;Koskela, J;Huang, H;Stevens, C;Pirinen, M;Haritunians, T;Neale, BM;Kurki, M;Ganna, A;Graham, D;Glaser, B;Peter, I;Atzmon, G;Barzilai, N;Levine, AP;Schiff, E;Pontikos, N;Weisburd, B;Lek, M;Karczewski, KJ;Bloom, J;Minikel, EV;Petersen, BS;Beaugerie, L;Seksik, P;Cosnes, J;Schreiber, S;Bokemeyer, B;Bethge, J;International IBD Genetics Consortium,;NIDDK IBD Genetics Consortium,;T2D-GENES Consortium,;Heap, G;Ahmad, T;Plagnol, V;Segal, AW;Targan, S;Turner, D;Saavalainen, P;Farkkila, M;Kontula, K;Palotie, A;Brant, SR;Duerr, RH;Silverberg, MS;Rioux, JD;Weersma, RK;Franke, A;Jostins, L;Anderson, CA;Barrett, JC;MacArthur, DG;Jalas, C;Sokol, H;Xavier, RJ;Pulver, A;Cho, $\mathrm{JH} ;$ McGovern, DPB;Daly, MJ

Title:

Insights into the genetic epidemiology of Crohn's and rare diseases in the Ashkenazi Jewish population.

Date:

2018-05

\section{Citation:}

Rivas, M. A., Avila, B. E., Koskela, J., Huang, H., Stevens, C., Pirinen, M., Haritunians, T., Neale, B. M., Kurki, M., Ganna, A., Graham, D., Glaser, B., Peter, I., Atzmon, G., Barzilai, N., Levine, A. P., Schiff, E., Pontikos, N., Weisburd, B. ,... Daly, M. J. (2018). Insights into the genetic epidemiology of Crohn's and rare diseases in the Ashkenazi Jewish population.. PLoS Genet, 14 (5), pp.e1007329-. https://doi.org/10.1371/journal.pgen.1007329.

\section{Persistent Link:}

http://hdl.handle.net/11343/273297

License:

CC BY 